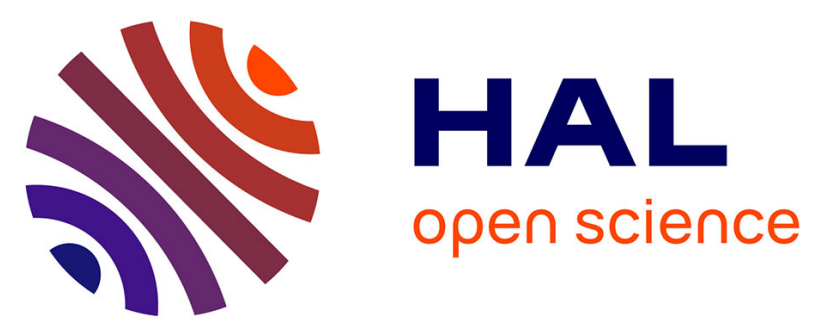

\title{
A rapid and low noise switch from RANS to WMLES on curvilinear grids with compressible flow solvers
}

\author{
Sébastien Deck, Pierre-Elie Weiss, Nicolas Renard
}

\section{To cite this version:}

Sébastien Deck, Pierre-Elie Weiss, Nicolas Renard. A rapid and low noise switch from RANS to WMLES on curvilinear grids with compressible flow solvers. Journal of Computational Physics, 2018, 363, pp.231-255. 10.1016/j.jcp.2018.02.028 . hal-01847366

\section{HAL Id: hal-01847366 https://hal.science/hal-01847366}

Submitted on 23 Jul 2018

HAL is a multi-disciplinary open access archive for the deposit and dissemination of scientific research documents, whether they are published or not. The documents may come from teaching and research institutions in France or abroad, or from public or private research centers.
L'archive ouverte pluridisciplinaire HAL, est destinée au dépôt et à la diffusion de documents scientifiques de niveau recherche, publiés ou non, émanant des établissements d'enseignement et de recherche français ou étrangers, des laboratoires publics ou privés. 


\title{
A rapid and low noise switch from RANS to WMLES on curvilinear grids with compressible flow solvers
}

\author{
Sébastien Deck ${ }^{\mathrm{a}, *}$, Pierre-Elie Weiss ${ }^{\mathrm{a}}$, Nicolas Renard $^{\mathrm{a}}$ \\ ${ }^{a}$ ONERA, The French Aerospace Lab, F-92190 Meudon, France
}

\begin{abstract}
A turbulent inflow for a rapid and low noise switch from RANS to Wall-Modelled LES on curvilinear grids with compressible flow solvers is presented. It can be embedded within the computational domain in practical applications with WMLES grids around three-dimensional geometries in a flexible zonal hybrid RANS/LES modelling context. It relies on a physics-motivated combination of Zonal Detached Eddy Simulation (ZDES) as the WMLES technique together with a Dynamic Forcing method processing the fluctuations caused by a Zonal Immersed Boundary Condition describing roughness elements. The performance in generating a physically-sound turbulent flow field with the proper mean skin friction and turbulent profiles after a short relaxation length is equivalent to more common inflow methods thanks to the generation of large-scale streamwise vorticity by the roughness elements. Comparisons in a low Mach-number zeropressure-gradient flat-plate turbulent boundary layer up to $R e_{\theta}=6100$ reveal that the pressure field is dominated by the spurious noise caused by the synthetic turbulence methods (Synthetic Eddy Method and White Noise injection), contrary to the new low-noise approach which may be used to obtain the low-frequency component of wall pressure and reproduce its intermittent nature. The robustness of the method is tested in the flow around a three-element airfoil with WMLES in the upper boundary layer near the trailing edge of the main element. In spite of the very short relaxation distance allowed, self-sustainable resolved turbulence is generated in the outer layer with significantly less spurious noise than with the approach involving White Noise. The ZDES grid count for this latter test case is more than two orders of magnitude lower than the Wall-Resolved LES requirement and a unique mesh is involved, which is much simpler than some multiple-mesh strategies devised for WMLES or turbulent inflow.
\end{abstract}

Keywords: Turbulent boundary layers, Hybrid RANS/LES methods, Zonal Detached Eddy Simulation, Wall-Modelled LES, Turbulent Inflow, Wall Pressure.

\section{Introduction}

\subsection{Context}

As discussed by Sagaut et al[86], multiscale and multiresolution approaches have attracted enormous recent interest in a variety of scientific disciplines and particularly in Computational Fluid Dynamics (CFD) where a wide range of applications from external aerodynamics to internal flows have to be covered. The classical approaches are RANS (Reynolds Averaged Navier-Stokes) and LES (Large Eddy Simulation or WRLES, Wall-Resolved Large Eddy Simulation, when solid walls are involved). The primary obstacle to practical LES of industrial flows involving attached boundary layers at high Reynolds

\footnotetext{
${ }^{*}$ Corresponding author

Email address: sebastien.deck@onera.fr (Sébastien Deck)
} 
number remains computational resources. A recent survey by NASA[101] estimates that WRLES for engineering purposes will still be out of reach even with 2030 leading HPC machines. In order to achieve a significant cost reduction, the near-wall dynamics has to be modelled while only the outer layer, e.g. $90 \%$ of the boundary thickness, is resolved [76]. The development of WMLES (Wall-Modelled Large Eddy Simulation) strategies is a necessity to remove the prohibitive WRLES grid requirements of near-wall turbulence in high Reynolds number turbulent flows as encountered in aeronautical applications (see discussions in $[104,19,29,27]$ among others). The present paper is primarily concerned with the practical use of WMLES-type methods.

The problem of zonal RANS/LES treatment is equivalent to a multiresolution decomposition and can take several forms, including turbulent inflow conditions, WMLES or embedded LES and results in a complex set of requirements. In this framework, the use of a zonal treatment of turbulence is therefore clearly advocated by the authors[26, 28, 27], because it provides an efficient framework to simulate accurately complex situations where only specific zones of the flow may require a high resolution specified and controlled by the user.

However, implementing advanced methodologies for wall turbulence simulations is a serious and still open issue. As an example, using the RANS field alone to generate inflow data for the LES domain cannot be sufficient: additional modelling which includes further assumptions on local length and time scales is required to recover "efficient" inlet conditions. In practice, a host of technically challenging issues arise. Hence, the expectations of turbulence generating methods intended for practical applications are discussed in the next section.

\subsection{Expectations of an efficient turbulent inlet method}

Boundary conditions play a dominant role for spatially inhomogeneous wall-bounded flows. The choice of the method to specify this inflow is problem-dependent and various methods have been proposed in the literature. We propose here to group them in four main families of methods (see table 1):

Family (a) Mapping/Recycling techniques

Within this class of methods, the idea consists either in generating inlet data from another simulation (precursor) or in dedicating a part of the computational domain to the use of a recycling method.

Family (b) Synthetic turbulence

Inflow methods relying on synthetic turbulence generation are based on the assumption that turbulence can be represented by a superimposition of coherent structures and thus can be specified by using only low-order statistics.

Family (c) Volume source terms

This kind of methods is based on the addition of a source term usually in the momentum equation. The intensity of this source term is classically proportional to the difference between the running time-average and a prescribed target value known from a RANS simulation for example.

Family (d) Controlled transition

These methods borrow ideas from the laminar/turbulent transition in the boundary layer. Either tripping or vortex generators induce large-scale turbulent eddies that initiate the cascade of turbulent kinetic energy from large to small scales. Numerical strategies based on theoretical linear stability analysis and optimal perturbations also belong to this family.

The main differences between these methods lie in the quality of the turbulence they generate, the computational distance, also called adaptation distance $L_{R}$, required to obtain a well-behaved turbulence, the extra cost they generate, 


\begin{tabular}{|c|c|c|c|c|c|}
\hline & $\begin{array}{c}\text { (a) Mapping } \\
\text { techniques }\end{array}$ & $\begin{array}{c}\text { (b) Synthetic } \\
\text { turbulence }\end{array}$ & $\begin{array}{c}\text { (c) Volumic } \\
\text { forcing }\end{array}$ & $\begin{array}{c}(d) \text { Controlled } \\
\text { transition }\end{array}$ & $\begin{array}{c}\text { Present } \\
\text { proposal }\end{array}$ \\
\hline Applicability to $3 D$ geometries & - & \pm & + & + & + \\
\hline Adaptation distance on $Q D N S / W R L E S$ grids & + & + & + & + & + \\
\hline$\left(L_{R}\right)_{Q D N S}$ & $\left(4-8 \delta_{0}\right)$ & $\left(4-8 \delta_{0}\right)$ & / & / & / \\
\hline Adaptation distance on $W M L E S$ grids & - & \pm & + & - & + \\
\hline$\left(L_{R}\right)_{W M L E S}$ & / & $\left(10-80 \delta_{0}\right)$ & / & / & $\leq 10-15 \delta_{0}$ \\
\hline Self - sufficiency/Initialisation & - & + & - & + & + \\
\hline Spurious noise & \pm & - & + & + & + \\
\hline Extension to highly compressible flows & + & \pm & / & 1 & / \\
\hline
\end{tabular}

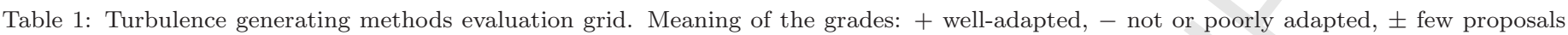

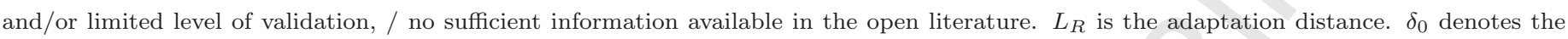
inlet boundary layer thickness.

the complexity of implementation of the method and initialisation of the calculation. Last but not least it is worth mentioning that most of the above methods have been assessed on canonical channel and flat plate boundary layer flows in the framework of DNS or WRLES grids but only few of them can be applied on three-dimensional curvilinear geometries and fewer yet have been assessed in a WMLES framework where their original efficiency can be significantly altered. These latter aspects have received poor attention in the literature, which may appear paradoxical since the development of advanced hybrid methods is precisely motivated by their potential capability to predict the fluctuating field in complex configurations. The problem becomes even more difficult if one is interested in fine details of the pressure or density fields (e.g. for aero-acoustics or aero-optics oriented studies).

Extensive reviews and hierarchical organizations of the various inflow generation methods can be found in several monographs $[86,40,114]$ and journal papers $([109,52,97,125])$ among others. We propose here to revisit some of these methods in terms of advantages and shortcomings according to the following two practical but highly-demanding criteria on both hydrodynamic and acoustic aspects respectively:

\section{- Criterion I : Applicability to 3D geometries and WMLES grids}

The shortness of the recovery or adaptation length, as well as the self-sufficiency of the initialisation of the calculation (by limiting the statistical information to be provided by the user), are important issues of a turbulence generating method intended to be used on curvilinear geometries.

Recycling methods based on the formulas of Lund et al.[60] are among the most effective and the least expensive inflow conditions available to simulate zero-pressure-gradient flat-plate boundary layer flows (see $[1,87,105,66]$ for extensions of the original method to pressure gradient or compressible flows as well as for limitations of the spurious frequencies induced by the periodic conditions). The adaptation distance lies classically between 4 and 8 initial boundary layer thicknesses $\delta_{0}$ on DNS and WRLES grids. In [31], a grid sensitivity in the framework of WMLES has been conducted with recycling methods and a relaminarisation has been observed for coarse grids. Hence, the use of recycling methods is by far not straightforward in a WMLES framework, as illustrated by the difficulties documented by Choi et al. [20]. Besides, another major difficulty relative to recycling methods remains their initialisation and their adaptation to relevant geometries (i.e. without any homogeneous direction) since both recycling and library lookup techniques may suffer from a lack of generality because they rely on self-similarity assumptions. Gloerfelt and Robinet [44] indicate also that these techniques suffer from a certain lack of robustness for long-term tracking, consistent with the findings of Sagaut et al. [87].

Compared to recycling methods, synthetic turbulence techniques are feed forward, making them a rapidly growing 
topic since most of the expectations for industrial simulations rely on such generation methods. Several ways to generate the random part have been proposed in the literature based on random Fourier mode synthesisation [97], scaling transformations of the Reynolds stresses[102, 4], digital filtering methods [53, 34, 126] or synthetic eddy methods [89, 47, 72] among others. Most synthetic turbulence methods can only provide an approximation of turbulence at the inlet so that the adaptation distance is usually longer than in the case of recycling approaches. Besides, Deck et al [32] have shown that with classical methods designed in an WRLES context (i.e. without fixing the RANS/LES interface height) the adaptation distance depends on the grid resolution and that very long transient can make WMLES not applicable in practice.

The use of source terms may accelerate the adjustment process towards equilibrium. Another asset of resorting to source terms comes from their ability to be directly introduced within a flow field instead of being reduced to an inflow boundary condition. This particular turbulence reactivation process allows the information to travel both ways across it, while synthetic turbulence generation methods or recycling ones impose a one-way turbulence injection. As a recent example Waindim and Gaitonde [117] used a counter blow body-force based approach to generate an equilibrium turbulent boundary layer suitable for DNS and WRLES simulations. The body-force induces a small separated region in an incoming laminar boundary layer that triggers transition to turbulence. In a different spirit, the strategy that involves body forcing addition in a closed-loop control was firstly introduced by Spille-Kohoff and Kaltenbach [106] and allows to act directly inside the flow and not only at the boundaries. The original formulation, also referred to as controlled forcing method in the literature, was successfully employed to reactivate a LES near-wall turbulent content on both simple canonical flows (see $[50,52]$ ) and applied configurations such as an afterbody flow [41], a transonic profile [84] or a high-lift configuration [128]. The reader has to be aware that in the previously mentioned zonal RANS/LES studies only a LES resolution in a wall-resolved sense is considered when employing the dynamic forcing method, without any near-wall RANS treatment. Especially, Laraufie et al [55] showed that, despite the relevance of the stimulation through body forces strategy, the original source term definition proposed in [106] fails in a WMLES context. These authors proposed a new source term which permits to minimize the transition distance in the framework of WMLES. This method was also successfully employed by Bocquet et al [9] in their study of a hot jet in a cross-flow. The Dynamic Forcing method alone is nevertheless not self sufficient since it has to be combined with another method that introduces initial velocity fluctuations as will be discussed in the following sections.

For the sake of completeness, let us also mention the methods based on ideas borrowed from the laminar/turbulent transition in a boundary layer (Family $(\mathbf{d}))$. Though this kind of methods is in principle more relevant to DNS than to WMLES issues, we will take advantage in the present work from some of their underlying ideas and theoretical results to accelerate transition from modelled to resolved turbulence.

Let us be reminded that laminar/turbulent transition can follow several routes [3]. Natural transition for a Blasius boundary layer on a perfectly smooth wall in a low free-stream disturbance environment results from the amplification of unstable waves (e.g. TS or Tollmien-Schlichting waves). In the presence of strong disturbances (high free-stream turbulence, large roughness elements), the role of the primary instability mechanism is bypassed and transient growth of non-normal mechanisms is another possible route to turbulence. Such non-normal mechanisms may also be identified in zero-pressure-gradient turbulent boundary layers with an eddy viscosity assumption in the linearized operator, although the turbulent mean profiles are linearly stable, i.e. there is no unstable mode, unlike in the laminar case. On the theoretical side, optimal perturbations (i.e. the most amplified, which are originated by streamwise vortices in these flows) and the associated transient energy growth sustained by turbulent boundary layers have been computed by Cossu 
et al. [22]. These authors have shown that the curves associated with the maximum energy growth display two optima. The first one is inner-scaled and is associated to a spanwise length scale $\lambda_{z}^{+} \approx 80\left(\lambda_{z}^{+}=\lambda_{z} / l_{\nu}\right.$ where $l_{\nu}$ is the viscous length scale) consistent with the streaky structures involved in the near-wall turbulence. The second optimum scales in outer units. Nevertheless these authors have shown that a wide range of spanwise wavelengths up to $\lambda_{z} / \delta \approx 30$ can be largely amplified. On the experimental side, Pujal et al.[79] used an array of suitably shaped cylindrical roughness elements to generate large-scale coherent streaks and suppressed flow separation at the rear-end of an Ahmed body. Duriez et al. [35] also used small cylinders as vortex generators to create streamwise counter-rotating vortices. Thanks to Particle Image Velocimetry (PIV), these authors have shown that a self-sustaining process is associated with the longitudinal destabilization of streamwise streaks. Similarly and thanks to tomographic PIV measurements, Ye et al [127] emphasized the importance of the front horseshoe vortex in the rapid transition to turbulence process.

To shorten the transition length in a numerical simulation, several methods have been proposed to reproduce a bypass scenario (see Gloerfelt and Robinet[44] for a recent review). As an example, Örlü and Schlatter[70] implemented tripping as a weak random volume force acting in the wall-normal direction and depending on both time fluctuations and random harmonic fluctuations in the spanwise direction. Common features are based on the introduction of long streaks of velocity perturbation and their lift-up from the wall results in an inflectional velocity profile which becomes sensitive to shortwavelength instabilities. Note that at this stage, only methods of Family (b) and (c) may satisfy the requirements of criterion $I$ since methods belonging to Family (a) and (d) are dedicated to DNS/WRLES studies.

\section{- Criterion II: Low spurious noise generation}

In many applications involving compressible flows, the acoustic waves are important and must be predicted accurately at the boundaries and in the interior. Garnier et al [40] reminded that no fully general solution which includes explicit reconstruction of fluctuations associated to each of the three Kovasznay modes is available in the open literature. The spurious acoustic waves that may be generated by boundary conditions can degrade the solution developing inside the domain [21].

Among the mapping techniques (Family (a)), let us mention the work by Viazzo et al[116] who proposed in a WRLES framework, a procedure to cancel the artificial pseudo-acoustic effect induced by the periodic boundary condition of a recycling technique.

Most of the current synthetic turbulence models (Family (b)) are designed to work with incompressible flow solvers, where the divergence-free condition is enforced. The velocity fluctuations induced by the imposed synthetic turbulence field generally does not satisfy continuity, so that the numerical solution tends to introduce significant pressure fluctuations close to the inlet in order to adapt the velocity field to the divergence-free condition. In the framework of incompressible flow solvers, Poletto et al [77] proposed a divergence-free synthetic eddy method (DFSEM) by working with the vorticity field. Similarly, Sescu and Hixon [93] construct a divergence-free synthetic eddy model in terms of a vector potential. It should however be emphasized that in a compressible flow, the velocity fluctuations are not necessarily divergence-free, and the treatment of the pressure field is fundamentally different in typical compressible and incompressible flow solvers. This makes the adaptation of divergence-free versions of SEM to compressible flow solvers nontrivial. Let us also be reminded that a sudden change in vortex strength can be a source of noise generation (vortex sound [78]). Hence the injection of vorticity at a turbulent inlet, for instance induced by a white noise, can be a source of spurious noise. Such a behaviour has been reported in the frame of other advanced synthetic turbulence methods (see [110]). As an example, in the frame of jet noise study, Shur et al[95] discourage the use of inflow perturbations arguing that it introduces many uncontrolled 
parameters in the simulation. To suppress the spurious noise induced by synthetic turbulence, the same authors [97] insert an internal damping layer in the LES subdomain. Though efficient on configurations featuring an homogeneous spanwise direction, its extension to three-dimensional geometries is not straightforward. Besides Colonius and Lele[21] remind that if buffer zone techniques are used then tunable parameters must be set through trial and errors.

To the authors' best knowledge, the effect of sole source terms (Family (c)) on the pressure field has been significantly less studied, probably because this kind of methods is generally not self-sufficient and needs to be associated with another turbulence generating method belonging to Family (a) or (b). In [28], the authors observe that no significant spurious noise is induced by the forcing itself and they argue that the dynamic forcing method acts as a source term in the momentum equation, but not on the velocity itself, contrary to a Dirichlet boundary condition which would be very noisy.

In the frame of methods belonging to Family (d), Gloerfelt and Berland[42] indicate that the use of steady obstacles has the great advantage of being stationary and produces considerably less spurious noise than the other inflow methods based on the introduction of unsteady perturbations. In the frame of WRLES of turbulent flat-plate boundary layers, Gloerfelt and Robinet[44] proposed a controlled transition from a base flow with an inflection point. The exponential growth of the resulting perturbation allows to add very weak disturbances limiting dramatically in return the spurious noise. Again, though very efficient for DNS/WRLES of canonical two-dimensional configurations, the extension of this class of methods to WMLES for three-dimensional applications is not straightforward.

According to our classification, the capabilities of methods belonging to Family (a), (b), (c) and (d) are gathered in table 1.

\subsection{Scope of the paper}

The previous short review (see table 1) reveals that though many techniques have been proposed in the literature to generate wall turbulence, none of them are self-sufficient or even trivial to carry out and all of them have limitations, approximations as well as assumptions associated with them. Importantly, most of the published methods have been applied on DNS or WRLES grids but only few of them have been assessed in the framework of WMLES with compressible flow solvers.

In this paper, we propose a new methodology based on the addition of source terms in the equations of motion as well as in the transport equation of the eddy/subgrid viscosity. More precisely, in $\S 2$ the salient features of our approach based on a physics-motivated combination of Zonal Detached Eddy Simulation (ZDES), Immersed Boundary Methods (IBC) and Dynamic Forcing (DF) are presented. The new method is then compared on both velocity and pressure fields with more classical synthetic turbulence methods on a spatially-developing flat-plate boundary layer in $\S 3$. The method is finally exercised on a more complex curvilinear geometry, namely a three-element airfoil in $\S 4$.

\section{Numerical method}

\subsection{General description of the numerical strategy}

In the frame of a finite volume approach, let us consider a finite volume $\Omega$ enclosed by a surface $\partial \Omega$ with $\mathbf{n}$ the unit outer normal surface vector associated to the surface element $d \Sigma$. The integral form of the compressible Navier-Stokes equations can be written as follows:

$$
\frac{\partial}{\partial t} \int_{\Omega} \mathbf{W} d \Omega+\oint_{\partial \Omega}\left(\mathbf{F}_{\mathbf{c}}[\mathbf{W}]-\mathbf{F}_{\mathbf{d}}[\mathbf{W}, \nabla \mathbf{W}]\right) \cdot \mathbf{n} d \Sigma=\int_{\Omega} \mathbf{T}(\mathbf{W}, \nabla \mathbf{W}) d \Omega
$$


where $\mathbf{W}$ is the conservative variable vector, $\mathbf{F}_{\mathbf{c}}$ and $\mathbf{F}_{\mathbf{d}}$ contain the convective and diffusive fluxes respectively and $\mathbf{T}$ denotes the source terms vector defined by:

$$
\mathbf{T}=\mathbf{T}_{Z D E S}^{(1)}+\mathbf{T}_{D F}^{(2)}+\mathbf{T}_{Z I B C}^{(3)}
$$

$\mathbf{T}_{Z D E S}, \mathbf{T}_{D F}$ and $\mathbf{T}_{Z I B C}$ denote respectively source terms associated to the ZDES turbulence modelling method and to the Dynamic Forcing method postprocessing the turbulent fluctuations generated by the ZIBC method (third source term). These three source terms are detailed in the following sections. The superscripts $\bullet^{(1)}, \bullet^{(2)}, \bullet^{(3)}$ indicate in what order the source terms are applied. The order of application of the terms is important in the inner iteration process (described in [121]).

\section{2. $T_{Z D E S}^{(1)}$ : Zonal Detached Eddy Simulation (ZDES)}

The Zonal Detached Eddy Simulation (ZDES) was first proposed by Deck [25] and the complete formulation that proposes an efficient solution to prevent delay in the formation of instabilities has been published in Ref. [26] (to which the reader is invited to refer for a full description). This hybrid RANS/LES method that belongs to the family of multiresolution approaches (see [86]) is initially based on the Spalart-Allmaras (SA) RANS model[103], characterized by the transport equation of a pseudo eddy-viscosity $\tilde{\nu}$ whose destruction term depends on the distance to the wall $d_{w}$. When the production and destruction terms of this model are balanced, the eddy viscosity scales with $d_{w}$ and the local vorticity magnitude $S, \nu_{t} \approx S d_{w}^{2}$.

Within ZDES, three specific hybrid length scale formulations entering the destruction term of $\tilde{\nu}$, also called modes, are then optimised to be employed on three typical flow field topologies (see table 2). Mode 1 concerns flows where the separation is triggered by a relatively abrupt variation in the geometry; mode 2 is retained when the location of separation is induced by a pressure gradient on a gently-curved surface and mode 3 for flows where the separation is strongly influenced by the dynamics of the incoming boundary layer. This latter mode is often referred to as Wall-Modelled Large Eddy Simulation (WMLES) mode (see Ref. [32]). This approach takes full advantage of its zonal nature, not only to allow the user to specify RANS and LES regions, but also to make possible the use of various formulations within the same calculation. Thus, ZDES offers an attractive flexibility in the treatment of turbulent flows in technical applications [27] including aeroacoustic studies [24]. In practice, within the ZDES framework, $d_{w}$ is replaced with $\tilde{d}_{Z D E S}$ in the pseudo eddy viscosity transport equation:

$$
\frac{\partial \rho \tilde{\nu}}{\partial t}+\nabla \cdot(\rho \tilde{\nu} \mathbf{u})=\mathcal{D}_{\tilde{\nu}}+T_{Z D E S}\left(d_{w}, \mathbf{W}, \nabla \mathbf{W}\right)
$$

where the diffusion and source terms read respectively as:

$$
\mathcal{D}_{\tilde{\nu}}=\nabla \cdot\left(\frac{1}{\sigma}(\mu+\rho \tilde{\nu}) \nabla \tilde{\nu}\right) \quad \text { and } \quad T_{Z D E S}=\frac{c_{b_{2}}}{\sigma} \nabla \tilde{\nu} \nabla(\rho \tilde{\nu})+\underbrace{\rho c_{b_{1}} \tilde{S} \tilde{\nu}}_{\text {production of } \tilde{\nu}}-\underbrace{\rho c_{w_{1}} f_{w}\left(\frac{\tilde{\nu}}{\tilde{d}_{Z D E S}}\right)^{2}}_{\text {destruction of } \tilde{\nu}}
$$

and the eddy viscosity is given by $\rho \nu_{t}=\rho \tilde{\nu} f_{v_{1}}$ and $\tilde{S}=S+\frac{\tilde{\nu}}{\kappa^{2} d_{w}^{2}} f_{v 2}$ where $S=|\nabla \times \mathbf{u}|$ is the vorticity magnitude. Functions $f_{v_{1}}, f_{v_{2}}$ and $f_{w}$ are near-wall correction functions while $\kappa, c_{b_{1}}, c_{b_{2}}, c_{w_{1}}$ and $\sigma$ are the original constants of the SA model[103]. The ZDES method aims to treat all classes of flow problems indicated in table 2 in a single model and has been successfully exercised by different research groups in different codes using finite volume solvers on both structured $[14,27,112]$ and unstructured grids [23] as well as finite element methods $[129,15]$ and finite differences [118]. The current 
paper is mainly concerned by mode 3 of ZDES as its WMLES operating mode which needs the definition of $d_{w}^{\text {interface }}$ detailed in $\S 3.2$ : (see table 2 )

$$
\tilde{d}_{Z D E S}=\tilde{d}_{D E S}^{I I I}=\left\{\begin{array}{ll}
d_{w} & \text { if } d_{w}<d_{w}^{\text {interface }} \\
\min \left(d_{w}, C_{D E S} \Delta_{v o l}\right) & \text { otherwise }
\end{array} \quad \text { where } \Delta_{v o l}=(\Delta x \Delta y \Delta z)^{1 / 3} \quad \text { and } C_{D E S}=0.65\right.
$$

with the RANS functions modified as $f_{v 1}=1, f_{v 2}=0, f_{w}=1$ if $d_{w}>d_{w}^{\text {interface. }}$ ZDES mode 3 has been used and validated for both WRLES [30, 81, 32] and WMLES [29, 55, 80, 46]. 
Zonal Detached Eddy Simulation (ZDES)

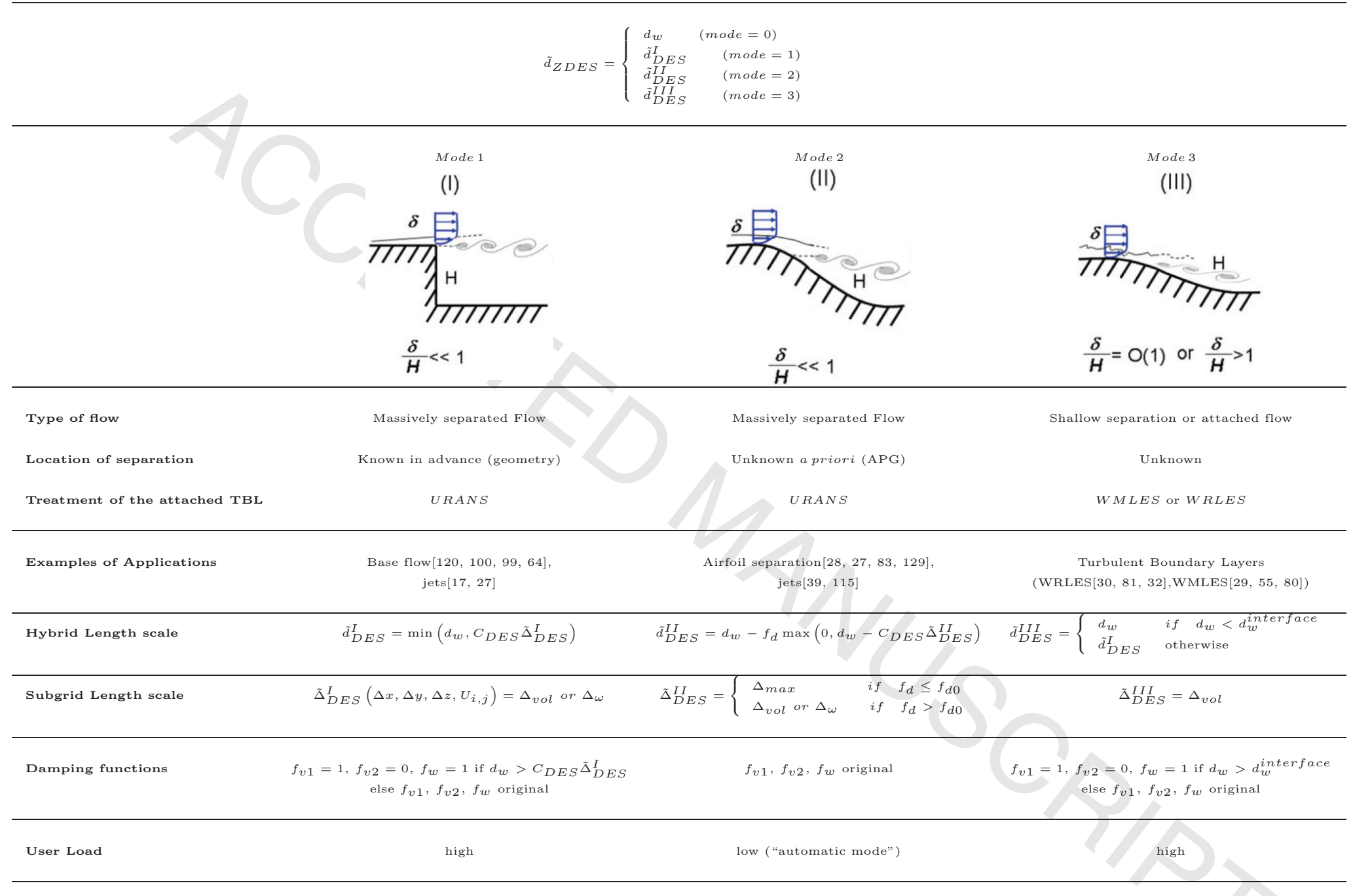

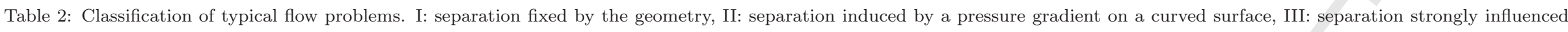

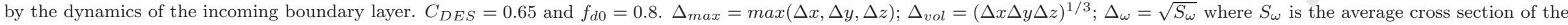
cell normal to the vorticity vector $\omega$ (see [26] for more details). 


\section{3. $T_{D F}^{(2)}$ : the DF Dynamic Forcing Method}

The turbulence reactivation process, retained for RANS to WMLES transitions, is based on work done by Laraufie et al [55] showing that the salient combination of a simple white noise with a dynamic forcing method (DF forcing in this paper) makes it possible to recover a self-sustainable well-behaved turbulent boundary layer on a short distance. For the sake of clarity, the principle of the method will be first presented (§2.3.1) in the framework of two-dimensionnal flat-plate boundary layer flows followed by its generalization to curvilinear grids in $§ 2.3 .2$.

\subsubsection{Principle}

The dynamic forcing approach aims to increase the resolved turbulent kinetic energy by adding a source term to the momentum equation which acts on a number of control planes downstream of the inlet to enhance wall turbulence activation and to reduce the required transition distance. This strategy was firstly proposed by Spille-Kohoff and Kaltenbach [106] who pointed out that, under flat plate boundary layer hypothesis, with $u, v$ and $w$ the velocity vector components defined respectively in the streamwise, wall normal and spanwise directions, the main contribution to the turbulent kinetic energy production is proportional to the Reynolds shear stress $\overline{u^{\prime} v^{\prime}}$, driven itself by the wall-normal Reynolds stress $\overline{v^{\prime} v^{\prime}}$. Indeed, it can be easily shown that under flat-plate boundary layer hypothesis, the main contribution to the Reynolds shear stress production is $-\overline{v^{\prime} v^{\prime}} \frac{d \bar{u}}{d y}$. This idea leads to the following formulation:

$$
\frac{\partial u_{i}}{\partial t}+\frac{\partial u_{j} u_{i}}{\partial x_{j}}=-\frac{1}{\rho} \frac{\partial P}{\partial x_{i}}+\nu \frac{\partial^{2} u_{i}}{\partial x_{j} \partial x_{j}}+\frac{1}{\rho} \frac{\partial \tau_{i j}^{t}}{\partial x_{j}}+\delta_{i 2} \underbrace{T_{D F}\left(u_{i}^{\prime}, \mathcal{E}\right)}_{\text {forcing source term }}
$$

The forcing term $T_{D F}$ is then a function of the local wall-normal fluctuating velocity and an error term $\mathcal{E}$ taken between a reference calculation (e.g. provided by RANS) and the unsteady running one. In their original paper, the forcing term proposed by Spille-Kohoff and Kaltenbach [106] can be considered as a Proportional Integral (PI) controller and $\mathcal{E}$ is defined as the error between the actual Reynolds shear stress and the target one. Importantly, the following conditions aim either to focus the action on the more energetic events, or to prevent from unrealistic large shear stress events:

$$
u^{\prime} v^{\prime}<0 ; \quad\left|u^{\prime} v^{\prime}\right|>0.0015 U_{0}^{2} ; \quad\left|u^{\prime}\right|<0.6 U_{0} ; \quad\left|v^{\prime}\right|<0.4 U_{0}
$$

Laraufie et al [55] showed that, despite the relevance of the stimulation through body forces strategy, the original source term definition proposed by Spille-Kohoff and Kaltenbach [106] fails in a WMLES context. The lag between a turbulent event and the forcing system reaction, also observed by Keating and Piomelli [51] when using a similar approach for a different purpose, was identified as the main problem. This led Laraufie et al.[55] to the introduction of a new source term which permits to minimize this transition distance and sets to zero the integral part of the PI controller. These authors proposed to directly resort to the wall-normal Reynolds stress at a given station $x_{0}$ rather than the Reynolds shear stress:

$$
T_{D F}\left(x_{0}, y, z, t\right)=\alpha \cdot \mathcal{E}\left(x_{0}, y, t\right) \cdot v^{\prime}\left(x_{0}, y, z, t\right) \quad \text { and } \quad \mathcal{E}\left(x_{0}, y, t\right)=\rho{\overline{v^{\prime}}}_{R A N S}^{z, t}-\rho{\overline{v^{\prime 2}}}_{Z D E S}^{z, t}
$$

The operator $\boldsymbol{\bullet}^{z, t}=\frac{1}{L_{z} T} \int_{0}^{L_{z}} \int_{0}^{T} \bullet d t d z$ used to evaluate mean quantities corresponds to the average in both time and spanwise (i.e. homogeneous) directions. A modelling approach of the wall-normal Reynolds stress using Boussinesq, Bradshaw et al [12] and Wilcox[122] assumptions based on the mean velocity field and RANS eddy viscosity is retained:

$$
{\overline{v^{\prime 2}}}_{R A N S}=\frac{2}{9} \cdot 2 k_{R A N S}, \quad k_{R A N S}=-\frac{{\overline{u^{\prime} v^{\prime}}}_{R A N S}}{0.3}, \quad-{\overline{u^{\prime} v^{\prime}}}_{R A N S}=\nu_{t}\left(\frac{\partial \bar{u}_{R A N S}}{\partial y}+\frac{\partial \bar{v}_{R A N S}}{\partial x}\right)
$$




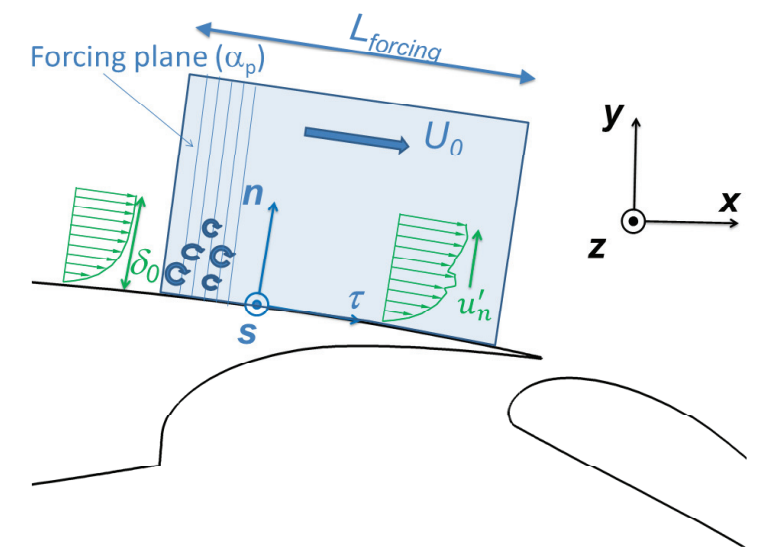

Figure 1: Sketch representing global and DF forcing coordinate systems.

The validation of the present modelling in the outer layer of the boundary layer over a wide range of Reynolds numbers in a WMLES context can be found in [54]. To assess ${\overline{v^{\prime 2}}}_{Z D E S}^{z, t}$, a time averaging process is carried out using an exponential window as proposed by Keating et al[52] in order to account for the flow evolution along time:

$$
\left.\overline{v_{Z D E S}^{\prime 2}} z, t+\Delta t\right)=\frac{\Delta t}{T_{a v g}} \overline{v_{Z D E S}^{\prime 2}} z=\left(1-\frac{\Delta t}{T_{a v g}}\right) \overline{v^{\prime 2}} z, t=(t)
$$

The window size is set up to $T_{a v g}=\frac{2 \delta_{0}}{U_{0}}$ where $\delta_{0}$ and $U_{0}$ denote respectively the initial boundary layer thickness and the local free-stream velocity. The spanwise average process is adopted to speed up the averaging process. Finally, only two parameters have to be set up by the user, namely:

- The forcing distance $L_{\text {forcing }}$ which has to be long enough so as not to experience turbulence relaxation at the end of the forcing area.

- The sum of the $\alpha$ parameters over the number of control planes $\left(\sum_{p=1}^{N p l a n e s} \alpha_{p}\right)$ which controls the forcing intensity.

\subsubsection{Extension to curvilinear grids}

The complete and detailed formulation of the DF forcing approach initially generalised in Ref. [28] to curvilinear structured grid codes is presented hereafter for the first time. Under boundary layer hypothesis, the direct relation that links the geometrical coordinate system $(\mathbf{x}, \mathbf{y}, \mathbf{z})$ to the dynamic forcing approach one, respectively the streamwise, wall normal and spanwise directions, leads to mathematical simplifications. One of them consists in restricting the application of the body force to the second momentum equation as illustrated in equation 6 . In the general case, the action of the forcing term (located in the dynamic forcing area as illustrated for example in fig. 3) is no more reduced to the second momentum equation but the components of the forcing term $\mathbf{T}_{\mathbf{D F}}$ affect the three momentum equations so that Eq. 6 becomes:

$$
\begin{gathered}
\frac{\partial\left(\rho u_{i}\right)}{\partial t}+\frac{\partial\left(\rho u_{j} u_{i}\right)}{\partial x_{j}}=-\frac{\partial P}{\partial x_{i}}+\frac{\partial\left(\tau_{i j}^{v}+\tau_{i j}^{t}\right)}{\partial x_{j}}+\rho T_{D F}\left(u_{n}^{\prime}, \mathcal{E}\right) n_{i} \quad \text { with } \\
T_{D F}(x, y, z, t)=\alpha \cdot \mathcal{E}(x, y, z, t)\left[\left(\mathbf{u}-\overline{\mathbf{u}}^{s, t i m e}\right) \cdot \mathbf{n}\right] \quad \text { and } \mathcal{E}(x, y, z, t)=\rho{\overline{u_{n}^{\prime 2}}}_{R A N S}^{s, t i m e}(x, y, z, t)-\rho{\overline{u_{n}^{\prime 2}}}_{Z D E S}^{s, t i m e}(x, y, z, t)
\end{gathered}
$$

Similarly to the $2 \mathrm{D}$ case $(\S 2.3 .1)$, the following conditions are imposed to focus the action on the more energetic events in the boundary layer $\left(u_{t}^{\prime} u_{n}^{\prime}<0,\left|u_{t}^{\prime} u_{n}^{\prime}\right|>0.0015 U_{0}^{2}\right)$ and to prevent from unrealistic large shear stress events 
$\left(\left|u_{t}^{\prime}\right|<0.6 U_{0},\left|u_{n}^{\prime}\right|<0.4 U_{0}\right)$. Note that $\alpha$ is not dimensionless since $\alpha \equiv\left[m . s . \mathrm{kg}^{-1}\right]$. Besides, the operator (noted $\left.\boldsymbol{\bullet}^{s, t i m e}\right)$ used to evaluate the mean quantities corresponds to the average in both time and spanwise (homogeneous) direction. This average process aims to speed up the convergence of the statistics when an homogeneous direction is present in the flow field. Otherwise in fully three-dimensional configurations (i.e. without any homogeneous direction), time average will be employed by itself $\left(\bar{\bullet}^{\text {time }}\right)$. The definition of the coordinate system, linked to the DF forcing application area, needs two salient pieces of information: the location of the wall, associated with the treated boundary layer, and the mean flow direction. However, both are already part of the data set required in the ZDES formulation dedicated to flat-plate boundary layers (see $[55,54]$ ). The wall location can be selected within the overall calculation boundary conditions, while the mean flow direction can be extracted from the RANS-SA flow field, used as reference when determining the dynamic forcing controller error function $\mathcal{E}(x, y, z, t)$ (see equation 12). This means that no additional effort is expected from the user when resorting to the present general dynamic forcing formulation. However, from the algorithm implementation side, the determination of the coordinate system basis $(\boldsymbol{\tau}, \mathbf{n}, \mathbf{s})$ is needed. As an example the wall-normal Reynolds stress ${\overline{u_{n}^{\prime 2}}}_{\text {RANS }}^{\text {stime }}$ can be assessed in a similar way to Eq. 9, namely:

$$
{\overline{u_{n}^{\prime 2}}}_{R A N S}^{s, t i m e}=\frac{2}{0.3} \cdot \frac{2}{9} \cdot \nu_{t} \cdot\left(\frac{\partial{\overline{u_{R}}}_{R A N S}^{s, t i m e}}{\partial n}+\frac{\partial{\overline{u_{n}}}_{\text {RANS }}^{s, t i m e}}{\partial \tau}\right)
$$

The DF forcing orthonormal basis, referred to as $(\boldsymbol{\tau}, \mathbf{n}, \mathbf{s})$, has then to be defined (see figure 1), which makes it possible to calculate body forces in $(\boldsymbol{\tau}, \mathbf{n}, \mathbf{s})$ while the DF forcing is effectively applied in the global $(\mathbf{x}, \mathbf{y}, \mathbf{z})$ coordinate system. Switching from one to another is done thanks to the Jacobian matrix of coordinate transformation:

$$
\mathbf{u}_{(\boldsymbol{\tau}, \mathbf{n}, \mathbf{s})}=\left(\begin{array}{ccc}
\frac{\partial x}{\partial \tau}\left(=\tau_{x}\right) & \frac{\partial y}{\partial \tau}\left(=\tau_{y}\right) & \frac{\partial z}{\partial \tau}\left(=\tau_{z}\right) \\
\frac{\partial x}{\partial n}\left(=n_{x}\right) & \frac{\partial y}{\partial n}\left(=n_{y}\right) & \frac{\partial z}{\partial n}\left(=n_{z}\right) \\
\frac{\partial x}{\partial s}\left(=s_{x}\right) & \frac{\partial y}{\partial s}\left(=s_{y}\right) & \frac{\partial z}{\partial s}\left(=s_{z}\right)
\end{array}\right) \cdot \mathbf{u}_{(\mathbf{x}, \mathbf{y}, \mathbf{z})}
$$

where the various components of $\tau_{(\mathbf{x}, \mathbf{y}, \mathbf{z})}, \mathbf{n}_{(\mathbf{x}, \mathbf{y}, \mathbf{z})}, \mathbf{s}_{(\mathbf{x}, \mathbf{y}, \mathbf{z})}$ are to be determined.

First of all, the wall normal vector $(\mathbf{n})$, solely geometry-dependent, is computed. At the wall, the normal vector $\left(\mathbf{n}_{\mathbf{w}}\right)$ of a given quadrilateral cell facet, defined in the coordinate system $(\mathbf{x}, \mathbf{y}, \mathbf{z})$ by $\mathrm{ABCD}$, is written as:

$$
\mathbf{n}_{w}=\frac{1 / 2(\mathbf{A C} \times \mathbf{B D})}{\|1 / 2(\mathbf{A C} \times \mathbf{B D})\|}
$$

Then, based on the consideration that DF forcing regions are restricted to attached boundary layers, an efficient method consists in spreading $\mathbf{n}_{w}$ out all along grid lines to the upper part of the forcing area assuming that the thickness of the boundary layer, when it is attached to the wall, is small compared to the wall curvature radius). Knowing the vector $\mathbf{n}$ field, the component $\boldsymbol{\tau}$ associated to the mean flow direction can be extracted from the reference mean flow field, also used in the controller term calculation. Furthermore, in order to ensure that $\boldsymbol{\tau}$ is perpendicular to $\mathbf{n}$, the component of the mean flow field in the wall normal direction is subtracted:

$$
\boldsymbol{\tau}=\frac{\overline{\mathbf{u}}_{R A N S}-\left(\overline{\mathbf{u}}_{R A N S} \cdot \mathbf{n}\right) \cdot \mathbf{n}}{\left\|\overline{\mathbf{u}}_{R A N S}-\left(\overline{\mathbf{u}}_{R A N S} \cdot \mathbf{n}\right) \cdot \mathbf{n}\right\|}
$$

Finally, $\mathbf{s}=\boldsymbol{\tau} \times \mathbf{n}$ completes the direct trihedron.

\section{4. $T_{Z I B C}^{(3)}$ : the Zonal Immersed Boundary Condition (ZIBC) method}

The introduction of streamwise vorticity feeding the dynamic forcing approach is performed using Zonal Immersed Boundary Conditions (ZIBC). The ZIBC formulation is inspired from the direct forcing approach as described by [65], 


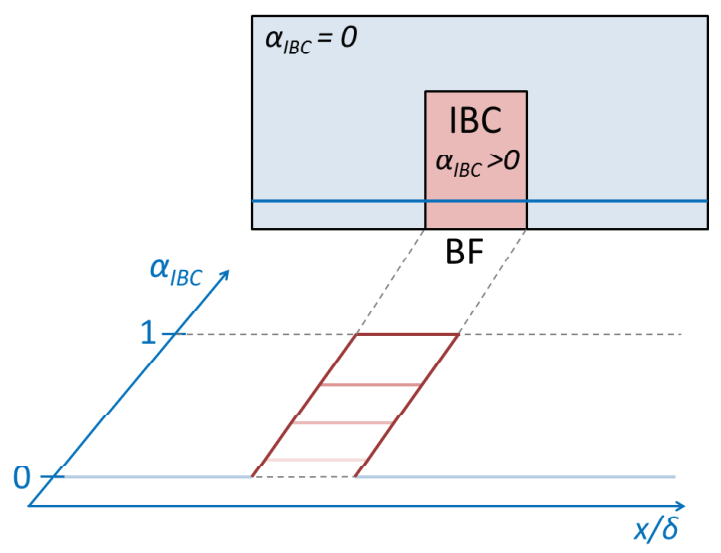

Figure 2: Sketch of the tag location used for the combined BF/IBC (Body-Fitted/Immersed Boundary Conditions) along with the streamwise distribution of $\alpha_{I B C} \in \mathbb{R}_{+}$.

[36] and [63] and adapted to the hybrid RANS/LES framework. These conditions are applied in the discrete form of the solved compressible Navier-Stokes equations through the use of the term $\mathbf{T}_{\mathbf{Z I B C}}^{(\mathbf{3})}$ which can be expressed as follows:

$$
\mathbf{T}_{\mathbf{Z I B C}}^{(\mathbf{3})}=\alpha_{I B C} \mathbf{f}_{\mathbf{I B C}}(\mathbf{W}, \nabla \mathbf{W})
$$

with $\alpha_{I B C}=0$ for a strictly fluid area and $\alpha_{I B C}=1$ for a strictly solid one. The forcing function $\mathbf{f}_{\mathrm{IBC}}$ is prescribed to reach the expected physical properties of the immersed boundaries by setting $\mathbf{f}_{\mathbf{I B C}}={ }^{t}\left(f_{\rho}, f_{\rho \mathbf{u}}, f_{\rho E}, f_{\rho \tilde{\nu}}\right)$ where $f_{\rho}, f_{\rho \mathbf{u}}$, $f_{\rho E}, f_{\rho \tilde{\nu}}$ stand for the forcing terms for the continuity, momentum, energy and Spalart-Allmaras transport equations, respectively. After a transient to reach the target values in the solid cells, the purpose of the ZIBC method is to turn the momentum and pseudo-eddy viscosity components of Eq. (1) locally into:

$$
\frac{\partial}{\partial t} \int_{\Omega} \mathbf{W} d \Omega=0
$$

so that after the initial transient, the momentum and pseudo-eddy viscosity components of the forcing term $\mathbf{T}_{Z I B C}^{(3)}$ can be read as:

$$
\int_{\Omega} \mathbf{T}_{Z I B C}^{(3)}(\mathbf{W}, \nabla \mathbf{W}) d \Omega=-\left[\int_{\Omega} \mathbf{T}_{Z D E S}^{(1)}(\mathbf{W}, \nabla \mathbf{W}) d \Omega+\int_{\Omega} \mathbf{T}_{D F}^{(2)}(\mathbf{W}, \nabla \mathbf{W}) d \Omega-\oint_{\partial \Omega}\left(\mathbf{F}_{\mathbf{c}}[\mathbf{W}]-\mathbf{F}_{\mathbf{d}}[\mathbf{W}, \nabla \mathbf{W}]\right) \cdot \mathbf{n} d \Sigma\right]
$$

The explicit expressions of the forcing terms (after the transient) are consequently as follows:

$$
\begin{gathered}
\left(f_{\rho}=0, \quad f_{\rho \mathbf{u}}=\nabla \cdot\left[\rho(\mathbf{u} \otimes \mathbf{u})+P \mathbf{I}-\left(\boldsymbol{\tau}^{v}+\boldsymbol{\tau}^{t}\right)\right]-\rho T_{D F} \mathbf{n}, \quad f_{\rho E}=0,\right. \\
\left.f_{\rho \tilde{\nu}}=\nabla \cdot\left[\rho \tilde{\nu} \mathbf{u}-\frac{1}{\sigma}(\mu+\rho \tilde{\nu}) \nabla \tilde{\nu}\right]-c_{b 1} \tilde{S} \rho \tilde{\nu}-\frac{c_{b 2}}{\sigma} \nabla(\rho \tilde{\nu}) \nabla \tilde{\nu}+\rho c_{w 1} f_{w}\left(\frac{\tilde{\nu}}{\tilde{d}_{Z D E S}}\right)^{2}\right)
\end{gathered}
$$

where $\boldsymbol{\tau}^{v}$ and $\tau^{t}$ correspond to the viscous and to the modelled turbulent stresses, respectively. The forcing term $T_{D F}$ (Eq. (11) and (12)) is set equal to zero outside of the dynamic forcing domain since $\alpha=0$ in this case.

Then, the mimicking of the solid boundary is obtained for non-zero values of $f_{\rho \mathbf{u}}$ and $f_{\rho \tilde{\nu}}$ whereas the continuity and energy equations remain unforced as in Mochel et al. [64] and Weiss et al. [121]. In particular, the term $f_{\rho}$ is kept equal to zero, which is an issue when the boundary is expected to strictly correspond to an adiabatic rigid wall changing the mass flow in a volume control surrounding the body modelled with IBC. On the contrary, in the present case, the zonal immersed boundary condition is used for a unique purpose which is the production of streamwise vorticity (a key 
feature of optimal perturbations to the mean turbulent profile ([22])), so that keeping the continuity equation unaltered constitutes an advantage because the flow should not be deviated upstream from the dynamic forcing zone. Then, the energy equation is also kept unchanged in order to avoid influencing locally and in an artificial manner the pressure levels of the flow field. Note that $\alpha_{I B C} \in[0,1]$ does not necessarily belong to the set of non-negative integers $\mathbb{N}$ but can be in the set of non-negative real numbers $\mathbb{R}_{+}$(fig. 2). As a consequence, the generation of streamwise vorticity can be obtained along with an attenuation of the presence of the obstacle to limit a backward-facing-step-type organization of the flow field. Nonetheless, this has not been investigated in this study where $\alpha_{I B C}$ simply is an integer.

\subsection{Summary}

The present method relies on a physics-motivated combination of ZDES, Dynamic forcing (DF) and the zonal use of IBC (ZIBC) previously described and implemented in the ONERA FLU3M research code. The code is written in Fortan 90 and solves on multiblock structured grids the compressible Navier-Stokes equations with a modified version of Liou's[59] AUSM+(P) scheme. This one proposed by Mary and Sagaut[62] integrates a wiggle detector which leads to a better control of the induced numerical viscosity. The time integration is carried out by means of an implicit second order accurate backward scheme. It uses an automatic domain decomposition and it fully exploits the Message Passing Interface (MPI) paradigm for the parallelism. The simulations are performed on 4-core Nehalem X5560 processors and the CPU cost per cell and per inner-iteration is about $3 \times 10^{-6} \mathrm{~s}$. Further details on the code can be found in [75, 121].

\section{Assessment of turbulent generation approaches}

\subsection{The flat-plate spatially developing turbulent boundary layer}

This first test case is a spatially developing zero-pressure-gradient turbulent boundary layer over a smooth flat plate. The free stream velocity is $U_{0}=70 \mathrm{~m} . \mathrm{s}^{-1}$, the static pressure is set to $P_{0}=99120 \mathrm{~Pa}$, the temperature equals $287 \mathrm{~K}$ leading to a Reynolds number per meter $R e=4.72 \times 10^{6} \mathrm{~m}^{-1}$ and a freestream Mach number $M_{0}=0.21$. These conditions are similar to those retained for the three-element airfoil investigated in $\S 4$. The initial boundary layer thickness is $\delta_{0}=5.8 \mathrm{~mm}$ so that the Reynolds numbers based respectively on the momentum thickness $\theta_{0}$ and the friction velocity $u_{*_{0}}$ at the inlet are respectively: $R e_{\theta}=\frac{U_{0} \theta_{0}}{\nu}=3040$ and $R e_{\tau}=\delta_{0}^{+}=\frac{u_{*_{0}} \delta_{0}}{\nu}=1065$.

The major parameters of the grid resolution are gathered in table 3 and correspond to classical grid resolutions used in the framework of the WMLES approach whose objective is to describe the outer part of the boundary layer. The first cell is at $y^{+}=1$ in the framework of a cell-centred code (i.e. the first vertex is at $y^{+}=2$ ). The grid distribution in the streamwise direction is not uniform in order to maintain $\Delta x / \delta \approx 0.1$ in the region of interest. Conversely, the grid in the spanwise direction is constant $\Delta z=\Delta x_{\min } / 2$ so that the total number of points is $N_{x y z}=7.7 \times 10^{6}$ points. Finally, the computational domain sizes in the streamwise, spanwise and wall-normal directions are respectively $L_{x}=113 \delta_{0}$, $L_{z}=5 \delta_{0}$ and $L_{y}=52 \delta_{0}$ so that the range of Reynolds numbers covered by the simulation is $3040 \leq R e_{\theta} \leq 6100$ (or $\left.1065 \leq R e_{\tau} \leq 2095\right)$. The physical time-step is set to $\Delta t_{C F D}=3.2 \times 10^{-7} \mathrm{~s}$ leading to $\Delta t^{+}=\frac{u_{*_{0}}^{2} \Delta t_{C F D}}{\nu}=0.15$ which satisfies the $\Delta t^{+}<1$ criterion proposed by Choi and Moin [18]. The simulation set-up is sketched in figure 3 which highlights the streamwise evolving RANS/LES interface $d_{w}^{\text {interface }}(x)$ as well as the turbulence generating method and eventually the dynamic forcing area that will be further detailed in the next section. 


\begin{tabular}{lrrcccc}
$\Delta x^{+}$ & $\Delta y^{+}$ & $\Delta z^{+}$ & $\Delta x / \delta$ & $\Delta z$ & $N_{x} \times N_{y} \times N_{z}$ & $N_{x y z}$ \\
\hline $100^{+}-200^{+}$ & 2 & 50 & $0.092-0.1$ & $\Delta x_{\text {min }} / 2$ & $587 \times 127 \times 103$ & $7.7 \times 10^{6}$
\end{tabular}

Table 3: Parameters of the grid. $N_{x}, N_{y}$ and $N_{z}$ are the grid sizes along the axes and the $\Delta$ 's are the corresponding resolutions expressed in both wall unit $\bullet+$ and inlet boundary layer thickness unit $\left(\delta_{0}\right)$.

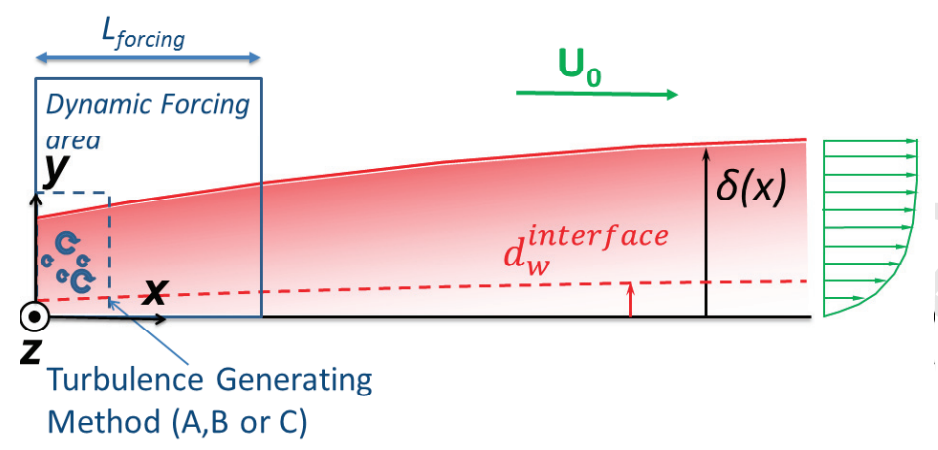

Figure 3: Simulation set-up in the case of the flat plate turbulent boundary layer. $\delta(x)$ is the local boundary layer thickness.

\subsection{Computational description}

Let us be reminded that the approach proposed in this paper is based on a combination of three main features (see Eq. 2) associating the WMLES approach (ZDES mode 3), the Dynamic Forcing (DF) method whose objective is to reprocess the velocity fluctuations provided by the turbulence generating method (relying on ZIBC). The performance of the new approach is compared with the ones of more classical inlet methods. Indeed, as soon as part of the boundary layer is resolved in LES mode, a realistic turbulent content has to be generated at the inlet of the domain to prevent decay of resolved turbulence which may lead to relaminarisation. Among synthetic turbulence methods, the SEM firstly proposed by Jarrin et al[47], improved by Pamiès et al [72] and adapted to WMLES in [32] is retained as a reference calculation named Case $A_{1}$ in the following. To assess the improvement brought by the Dynamic Forcing method, Case $A_{2}$ associates both SEM and DF features (see table 4).

While the DF approach was first [55] combined with the SEM method, its ability to lead a simple White Noise (WN) to a self-sustainable well-behaved boundary layer was assessed by Laraufie et al [56], who argue that SEM can be considered as an advanced turbulent inflow method, much more complex to implement than a simple white noise. Hence, with regards to practical applications, they propose in the frame of the DF method to add a simple anisotropic white noise over three computational cells in order not to be immediately annihilated by numerical dissipation as would be the case if the fluctuations were imposed over only one cell. In practice, a uniform random series $\varepsilon(t)=[-1,1]$ with $\bar{\varepsilon}^{t}=0$ and

\begin{tabular}{lcccc} 
& Case $A_{1}$ & Case $A_{2}$ & Case $B$ & Cases $\left(C_{i}\right)_{i=1 . .4}$ \\
\hline Inlet fluctuations generator & $S E M$ & $S E M$ & $W N$ & $Z I B C$ \\
Dynamic Forcing (DF) & No & Yes & Yes & Yes
\end{tabular}

Table 4: Definition of the inflow treatment cases for the flat-plate turbulent boundary layer. 


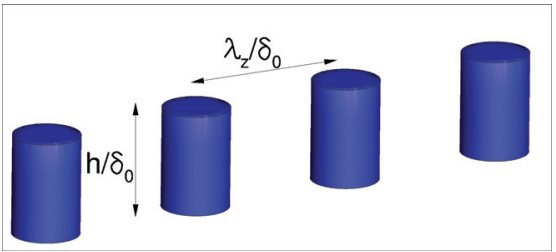

\begin{tabular}{lcccc} 
& Case $C_{1}$ & Case $C_{2}$ & Case $C_{3}$ & Cases $C_{4}$ \\
\hline$\lambda_{z} / \delta_{0}$ & 1 & 1 & 0.5 & 0.5 \\
$h / \delta_{0}$ & 0.6 & 0.3 & 0.3 & 0.6
\end{tabular}

Table 5: Shape of the roughness elements and relevant parameters for the $C_{i}$ 's calculations.

$\sigma_{\varepsilon}^{2}=1 / 3$ is generated. The instantaneous fluctuating velocity is then defined as:

$$
\mathbf{u}_{(\boldsymbol{\tau}, \mathbf{n}, \mathbf{s})}^{\prime}(t)={ }^{t}\left(\begin{array}{lll}
4.3 & 1.4 & 1.8)
\end{array} \frac{U_{0}}{100} \varepsilon(t)\right.
$$

The fluctuating velocity intensities given in the brackets are set-up in order not to exceed the maximum of the normal Reynolds stresses classically observed in a flat plate turbulent boundary layer. The calculation combining the white noise and the Dynamic Forcing is referred to as Case $B$ in the following (see table 4 ).

Calculations based on Cases $C_{i}$ are based on a completely different spirit and take advantage of the theoretical findings by Cossu et al[22] on optimal perturbations and transient energy growth sustained by a turbulent boundary layer. On the experimental side, Pujals et al [79] used an array of cylindrical roughness elements to generate nearly optimal vortices. In a similar way, such elements are immersed at the inlet of the computational domain thanks to the ZIBC approach. The relevant parameters of the roughness elements are the height of the cylinders $h / \delta_{0}$ and the spanwise spacing $\lambda_{z} / \delta_{0}$. These parameters are illustrated in table 5. Though Cossu et al. [22] have shown that a wide range of spanwise wavelengths up to $\lambda_{z} / \delta \approx 30$ can be largely amplified, due to the width of the computational domain $\left(L_{z} / \delta_{0}=5\right)$ only "limited" spanwise spacings $\left(\lambda_{z} / \delta_{0}\right)$ are studied. Table 5 summarizes the different $C_{i}$ configurations investigated in this study.

To be complete, we still have to specify the relevant parameters of the dynamic forcing and WMLES methods. As regards the two parameters of the DF approach (see $\S 2.3 .1$ ), similar values as the ones retained by Laraufie et al[55] are used here for all calculations, namely $L_{\text {forcing }}=7 \delta_{0}\left(N_{\text {planes }}=70\right.$ cells in the streamwise direction $)$ and $\sum_{p=1}^{N_{\text {planes }}} \alpha_{p}\left(\rho U_{0} \delta_{0}\right)=$ 29000. Note that these parameters are not optimal since Laraufie et al[55] indicate that the tuning of $\Sigma \alpha$ is case-dependent and these authors propose an optimization process. Nevertheless, the choice of not optimizing the case-dependent parameters allows to assess the robustness of the method which is an important outcome for the use in an industrial context. Concerning the WMLES method, the ZDES mode 3 switching into LES mode is presently prescribed by the user at the altitude $d_{w}^{\text {interface }}(x)=0.125 \delta(x)$ (Eq. (5)) which has a constant outer-scaled height, i.e. it evolves proportionally to the local boundary layer thickness $\delta(x)$ (see $[29,80]$ for further discussion of the interface location). $\delta(x)$ is determined by a precursor RANS calculation.

\subsection{Instantaneous flowfield}

An overview of the turbulent content generated by the ZDES simulations is evidenced in figure 4 by showing the iso-surfaces of the $\mathrm{Q}$ criterion $\left(Q=\frac{1}{2}\left(\|\boldsymbol{\Omega}\|^{2}-\|\mathbf{S}\|^{2}\right)\right.$ with $\mathbf{S}$ and $\boldsymbol{\Omega}$ denoting respectively the strain rate and rotation rate tensors). In the framework of the SEM method, without any forcing the coherent structures development is clearly delayed. One can indeed see the structures appearing much closer to the inlet when a volumic forcing (case $A_{2}$ ) is applied than when no forcing (case $A_{1}$ ) is applied. The efficiency of the DF forcing is further demonstrated with case $B$ showing that the method is able to reprocess the disturbances excited by a basic white noise. Indeed, turbulent structures appear close to the inlet $\left(\approx 3 \delta_{0}\right)$ and a heavy population of coherent structures appears almost instantaneously when the boundary 
layer develops further downstream. This major achievement was first shown in [56] on flat plate calculations and in [28] on a curvilinear configuration at a slightly lower Reynolds number $\left(R e_{\theta} \approx 3000\right)$. Let us be reminded that one of the most significant aspects of turbulence is its spatial and temporal coherence in the long wave range quantified by the integral and Taylor scales. When simply superimposing random noise on the inlet mean velocity profile the flow usually relaminarises quickly downstream the inlet [92]. The random noise inlet conditions suffer from the lack of correlation in both time and space. As a result, these random fluctuations lack usually in the low-wave number part of the kinetic energy spectrum and are very quickly dissipated (especially on coarse grids typical of WMLES), without sustaining real turbulence. The improvements come from the conditional imposition of the source term given by Eq 7 which allows to focus the action of the forcing term on the more energetic events and which can be considered as a way to re-introduce the lacking phase information.

The efficiency of the DF method to reprocess the incoming velocity fluctuations is confirmed by the different $C_{i}$ 's cases where a well behaved turbulent boundary layer is also developing quickly from the inlet. The basic flow about an isolated 3D element consists of a steady horseshoe vortex wrapped around the upstream side of the obstacle, with two steady counter-rotating legs trailing downstream (figure 4(h). See also [107] for a detailed discussion of the flow organisation around an isolated roughness element). These steady disturbances evolve rapidly downstream into low- and high-speed streaks aligned with the flow direction. Ye et al [127] used tomographic PIV to investigate boundary layer transition over isolated roughness elements of different geometry (cylinder, square, hemisphere and micro-ramp). They observed minor differences in terms of streamwise evolution of velocity fluctuations and streak amplitude between the bluff-front elements (cylinder, square and hemisphere). These latter elements induce a horse-shoe vortex due to upstream flow separation, leading to more rapid transition than the slender micro-ramp. In other words, the horseshoe vortex whose legs induce sideward low-speed regions in the wake appears to be the common denominator among all geometries considered. In practice this result shows that the exact form of the bluff-front elements is not crucial, which permits to use a ZIBC discretisation as described in $\S 2.4$.

In addition to the turbulent content, a first glimpse of the acoustic efficiency of the different inlet methods can also be gained by considering the density time derivative field. In the framework of calculations $A_{1}, A_{2}$ and $B$ using synthetic turbulence, the acoustic field is clearly dominated by intense spurious sound waves originating and having their highest amplitude along the inlet boundary. As could be anticipated, case $B$ using white noise at the inlet features smaller wave lengths than the cases $A_{i}$ using SEM where large scale structures are prescribed at the inlet. The velocity fluctuations induced by the imposed synthetic turbulence (SEM or random noise) generally do not satisfy the continuity equation. As a consequence, the numerical solution tends to introduce significant pressure fluctuations close to the inlet in order to adapt the velocity field to the continuity equation. Besides, let us be reminded (see $§ 1$ ) that the sudden change of vorticity levels induced by the white noise can also be a severe source of spurious sound generation (vortex sound [78]). Such a behaviour is not specific to the use of white noise and has been reported in the frame of other more sophisticated synthetic turbulence methods [110, 72, 97]. Besides, no major difference on the density time derivative field can be depicted between case $A_{1}$ and $A_{2}$ indicating that the DF forcing does not itself create significant noise (this had already been highlighted in $[28])$.

This latter major comment is confirmed by the $C_{i}$ configurations which feature a radical weakening if not a complete disappearance of these spurious sound waves. As reminded in the introduction, the methodology used for the $C_{i}$ configurations has the great advantage of being steady and produces considerably less spurious noise than the other inflow methods 


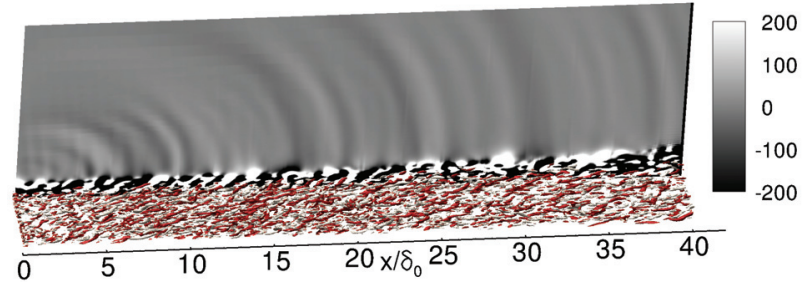

(a) case $A_{1}$

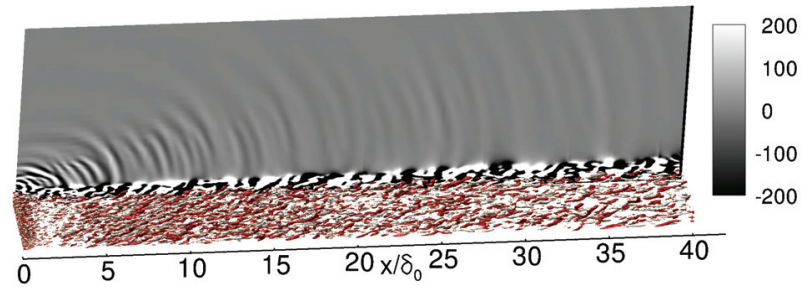

(c) case $B$

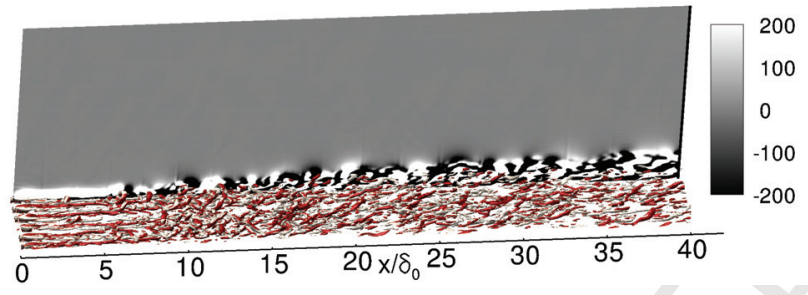

(e) case $C_{2}$

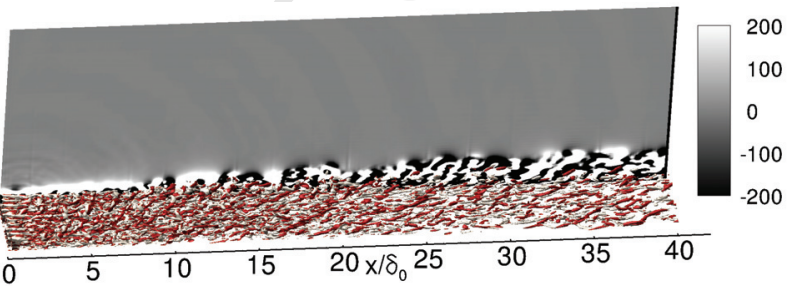

(g) case $C_{4}$

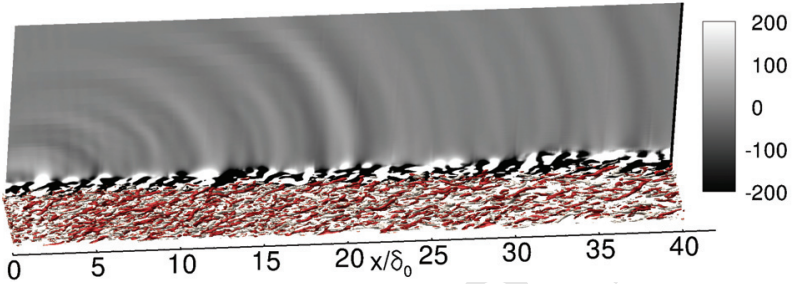

(b) case $A_{2}$

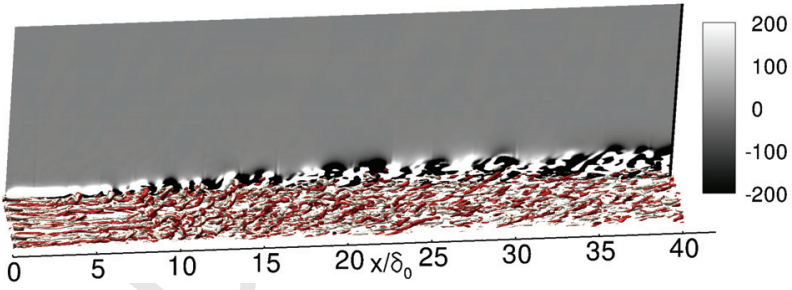

(d) case $C_{1}$

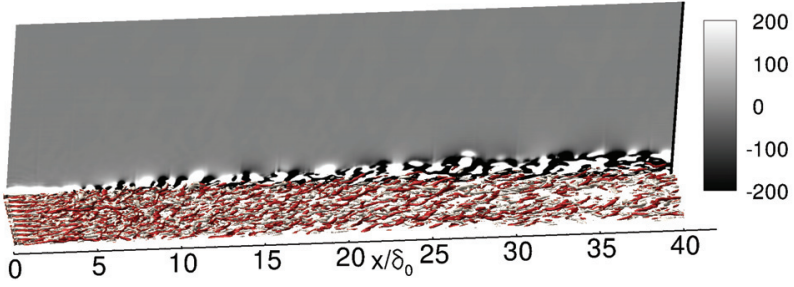

(f) case $C_{3}$

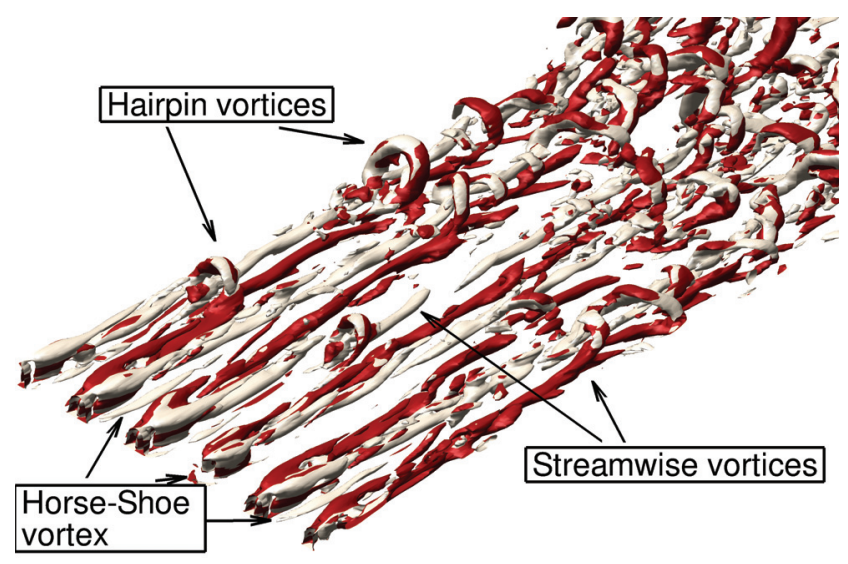

(h) Zoom near inlet of case $C_{2}$

Figure 4: Iso surface of the $\mathrm{Q}$ criterion $Q=0.25 U_{0}^{2} / \delta_{0}^{2}$ coloured by the streamwise component of vorticity $\left(\omega_{x}<0\right.$ in red and $\omega_{x}>0$ in white). Instantaneous field of $-\frac{\partial \rho}{\partial t}$ (gray scale colormap). 


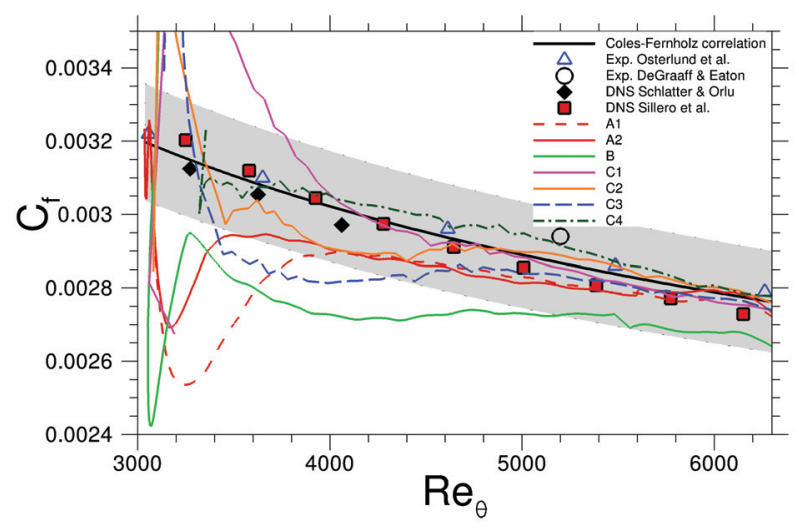

(a) $C_{f}\left(\operatorname{Re}_{\theta}\right)$

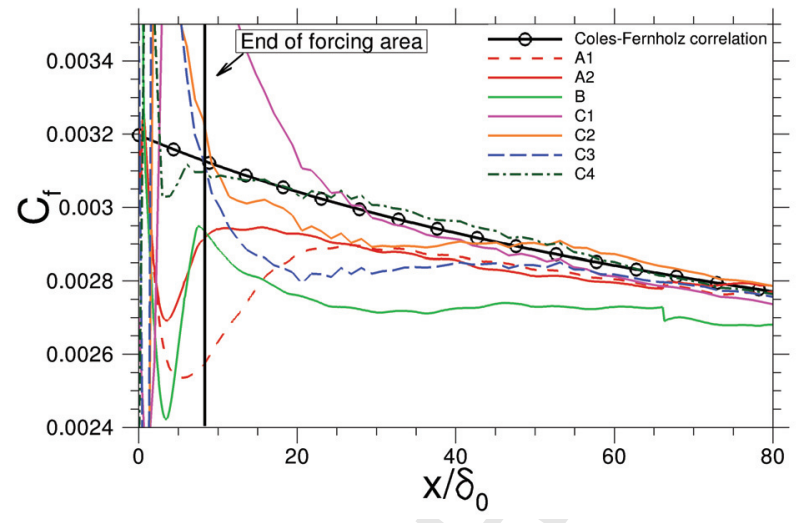

(b) $C_{f}\left(x / \delta_{0}\right)$

Figure 5: Streamwise evolution of the skin friction coefficient. The shaded area depicts a $5 \%$ tolerance margin with respect to the Coles-Fernholz correlation $C_{f}^{C F}=2\left(\frac{1}{0.384} \ln \left(R e_{\theta}\right)+4.127\right)^{-2}$.

based on the introduction of unsteady velocity perturbations. A deeper quantitative investigation of both velocity and pressure fields is now proposed in $\S 3.4$ and 3.5 respectively.

\subsection{Reynolds averaged data}

The skin friction coefficient $C_{f}$, defined as $C_{f}=\frac{\tau_{w}}{\frac{1}{2} \rho U_{0}^{2}}=2\left(\frac{u_{*}}{U_{0}}\right)^{2}$, constitutes a primary quantity of interest. In addition to several datasets available in the literature including both DNS and experimental data ([71, 33, 91, 98]), numerical results can also be compared with the widely acknowledged Coles-Fernholz correlation calibrated by Nagib et al[67]. The skin friction is compared for all calculations with these data in figure 5 . Except case $B$ resorting to simple white noise at the inlet, all simulations are within a $5 \%$ tolerance margin about the Coles-Fernholz correlation for Reynolds numbers higher than $R e_{\theta}=4500$. The outcome concerning case B partially results from the lack of large-scale structures at the inlet. Indeed based on a spectral analysis of the FIK[38] idendity, Deck et al [30] have shown that structures with a streamwise wavelength $\lambda_{x}>\delta$ contribute to more than $50 \%$ of the skin friction (see [82] for a theoretical discussion of the $C_{f}$ coefficient generation). Even on WMLES type grids, these authors have shown that the modelled $C_{f}$ amounts to only a bit more than $10 \%$ of the total $C_{f}$, indicating that most of the skin friction is resolved by WMLES rather than modelled. In the framework of calculations $A_{i}$ and $C_{i}$, large scales are explicitly injected (Cases $A_{i}$ ) or generated (cases $C_{i}$ ) at the inlet leading to significant improvement of the $C_{f}$ assessment compared with case $B$. In particular, case $C_{2}$ stays within the $5 \%$ tolerance margin about the Coles-Fernholz correlation as soon as the forcing area is ending, i.e. after a relaxation distance approximately coinciding with $L_{\text {forcing }}=7 \delta_{0}$.

The mean velocity profiles as well as the resolved normal Reynolds stresses are plotted in figure 6. Figure 6(a) indicates that the profile of the wake layer is well captured by the coarse mesh simulations, and so is the inner layer velocity profile. As classically observed in the framework of WMLES simulations on coarse grids ([96, 20]), a slight Log-Layer Mismatch (LLM) located near the RANS/LES interface can be depicted. The detailed discussion of the LLM issue is beyond the scope of this article but let us mention that no general, definitive and theory-motivated hybrid RANS/LES solution for curvilinear geometries has been published yet (see Shur et al. [96] and Shen and Edwards [94]). It is observed in Ref. [29] that this mismatch significantly decreases at a higher Reynolds number. 


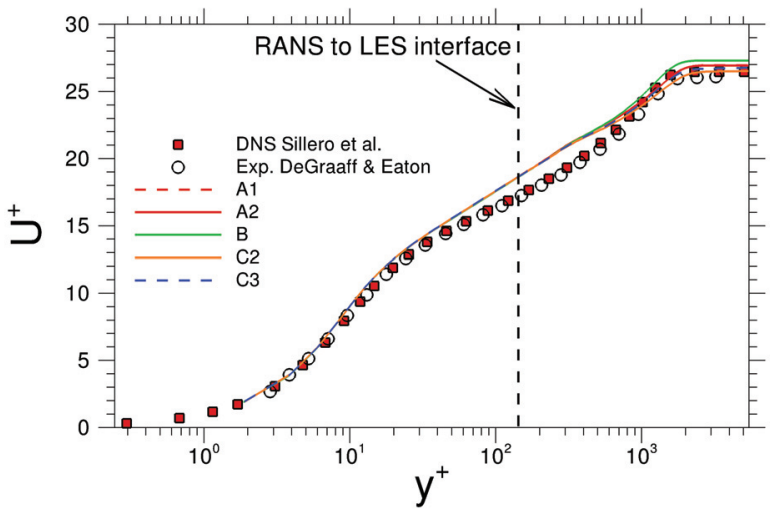

(a) Mean velocity profile

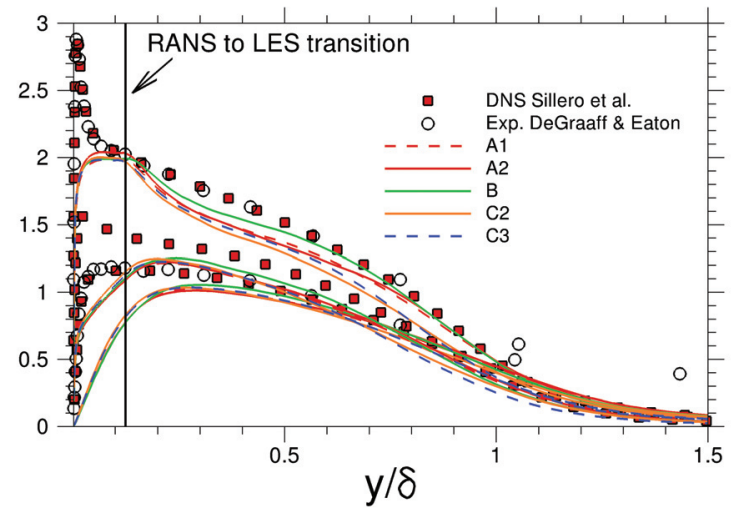

(b) Resolved normal Reynolds stresses $\left(u_{r m s}^{+}, v_{r m s}^{+}, w_{r m s}^{+}\right)$

Figure 6: Reynolds-averaged data at $R e_{\theta}=5200$

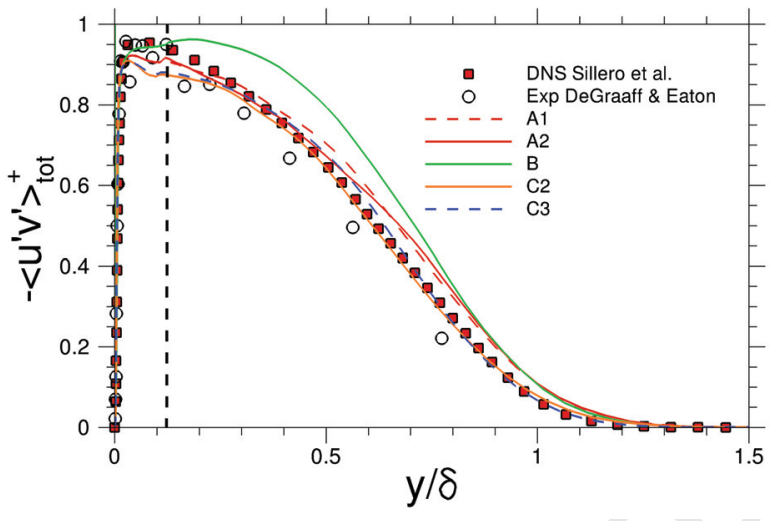

(a) Total Reynolds shear stress (both resolved and modelled)

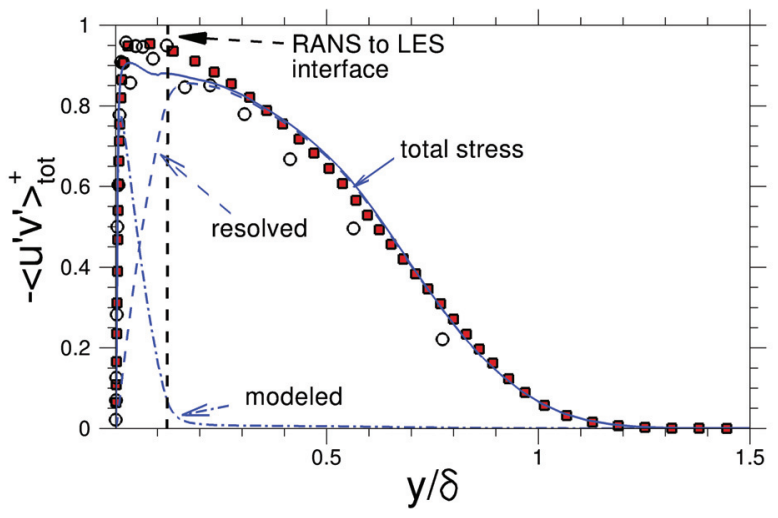

(b) Decomposition of the total Reynolds shear stress into resolved and modelled parts on configuration $C_{3}$

Figure 7: Reynolds shear stress at $\operatorname{Re}_{\theta}=5200$

The normal Reynolds stresses from the ZDES simulations are also compared in figure 6(b) with DNS (Sillero et al.[98] at $R e_{\theta}=5500$ ) and experimental data (DeGraaff \& Eaton[33] at $R e_{\theta}=5200$ ). The location of the RANS/LES interface is indicated since comparing the normal Reynolds stresses for $y<y^{\text {interface }}$ is meaningless. As already emphasized in Ref. [32, 29], the RANS/LES interface does not act as a turbulence barrier and is permeable to fluctuations since no sudden jump of the RMS quantities is noticed. One may also note that there is a significant level of unsteady fluctuations even within the (U)RANS zone, i.e. below the RANS/LES interface, especially close to the interface. The initial goal of WMLES however is not to resolve the inner layer dynamics, so that a linear scale has been chosen for figure 6(b) in order to put the emphasis on the outer layer. The streamwise turbulent intensity $u_{r m s}^{+}$is slightly underestimated at an intermediate height in the outer layer for all calculations. It is also worth noting that results for calculations $A 1$ and $A 2$ are identical. These results are consistent with the findings of Laraufie et al.[55] since these authors have demonstrated that no spurious mark is left within the aerodynamic flow field downstream the DF forcing application region. 
The total Reynolds shear stress, which is the sum of the resolved stress and the modelled stress, is compared with DNS and experimental values in figure 7(a). The total Reynolds shear stress magnitude is generally well predicted in the outer layer except for calculation $B$ which has not reached a full equilibrium state at the considered station. The lack of total shear stress near the RANS/LES interface is discussed in [29]. Indeed, the velocity fluctuations normal to the wall are underpredicted in the outer layer close to the interface while the streamwise fluctuations keep high levels, and are even overpredicted in places. This implies that the observed decrease of the resolved Reynolds shear stress near the interface is caused by the normal fluctuations, which do not penetrate easily into the RANS inner zone $\left(i . e\right.$. for $\left.y<d_{w}^{\text {interface }}\right)$.

Both modelled and resolved Reynolds stresses are then plotted for case $C_{3}$ in figure $7(\mathrm{~b})$. This figure reveals once again how the resolved fluctuations penetrate deeply into the inner layer treated in RANS mode, where they are only gradually replaced by the modelled Reynolds shear stress as one gets closer to the wall. It should also be noted that in the outer region treated in LES mode, the contribution of the modelled shear stress is negligible, except in the very vicinity of the interface, where the fluctuations seem to be damped by the interface and replaced by some modelled shear stress.

\subsection{Pressure field}

The pressure fluctuations, which are difficult to obtain from experiments (see discussion in Ref. [5]) because of the size of the transducer and the existence of a wide range of excited scales, deserve some discussion. First, let us be reminded that pressure is a non-local variable which provides a footprint of the turbulent boundary layer structures. In other words, the analysis of the pressure fluctuations in the framework of WMLES of wall-bounded flows is by far not trivial, rarely addressed or even shown in the literature and most published numerical studies concern mainly DNS or highly resolved WRLES simulations $[7,42,7]$. In this section, the performances of configurations $A, B$ and $C$ are further investigated. To this end, it is important to emphasize that the objective is not to perform an aeroacoustic study of the noise generated by a boundary layer but to assess the performance of the present new method in terms of spurious noise generation at the inlet and its consequences further downstream. The resolution of wall-bounded turbulence can indeed be necessary for aeroacoustic studies other than the prediction of the turbulent boundary layer noise itself. For instance, the prediction of trailing edge noise can be improved if the dynamics of the turbulent boundary layers is resolved with no spurious noise generation ([124]) instead of resorting to empirical models. Another example is the study of jet noise for which the incoming turbulence from the boundary layers at the nozzle exit may be determining ([13]).

First, the drastic positive effect on the noise provided by the configurations $C_{2}$ and $C_{3}$ is highlighted in figure 8 which shows the streamwise evolution of the sound pressure level as a function of the distance to the inlet at a constant altitude $\left(y / \delta_{0}=12.5\right)$ located outside the boundary layer. No difference between cases $C_{2}$ and $C_{3}$ is observed, which can be considered as a positive outcome concerning the robustness of the method as regards the pressure field. It is evidenced that the present synthetic turbulence method (configuration $A$ ) is the one that generates the most important noise at the inlet. As already seen in figure 4, the dynamic forcing method does not itself modify the properties of the pressure field (see configurations $A 1$ and $A 2$ ). While SEM (case $A$ ) injects energetic large-scale structures, case $B$ based on the use of single white noise (i.e. small scales) is significantly more silent at the inlet as well as further downstream. Unlike SEM, the white noise produces only short wave lengths which are quickly dissipated by viscosity and scattered by turbulence.

In the framework of spanwise-periodic calculations, the possible spurious noise sources induced by the inlet methods may be viewed in the farfield as a line of coherent sources. Bies and Hansen[8] indicate that a line of coherent sources which are in phase with one another radiates in the farfield like a continuous infinite line source of cylindrical waves. At the 


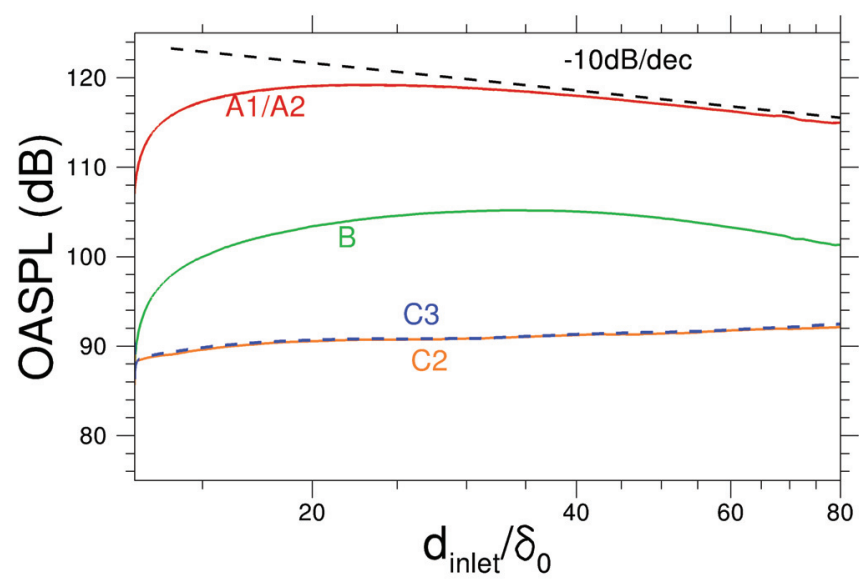

Figure 8: Streamwise evolution of $O A S P L=20 \log _{10}\left(\frac{P_{r m s}}{2 \cdot 10^{-5}}\right)$ with the distance to the inlet $d_{\text {inlet }} / \delta_{0}$ at constant altitude $y=12.5 \delta_{0}$.

point of observation located at the distance $d_{\text {inlet }}$ from the source line, the cylindrical waves have a surface area per unit span equal to $2 \pi d_{\text {inlet }}$, so that the mean square sound pressure at the point of observation scales as $<p^{2}>\propto \frac{1}{d_{\text {inlet }}}$. Thus the corresponding sound pressure level decreases as $-10 \log _{10}\left(d_{\text {inlet }}\right)$ (see figure 8 ). Of interest, one can note that only configurations $C$ feature an increase of pressure fluctuations outside the boundary layer as $R e_{\theta}$ increases which suggests that these fluctuations are not dominated by the artefacts of the inlet.

The wall-normal distributions of rms values of pressure $P_{r m s}^{+}=P_{r m s} / \tau_{w}$ at station $R e_{\theta}=5200$ are reported in figure 9 (a) and compared with the incompressible DNS data by Sillero et al[98]. Such a comparison of $P_{r m s}$ obtained with the present compressible flow solver is justified. Indeed, Bernardini and Pirozzoli [7] argued that the structure of the wall pressure field is very weakly affected by compressibility effects (at least for free-stream Mach numbers lower than 4) since the effect of the sound mode is quite limited while the vorticity mode provides a dominant contribution. In cases $A$ and $B$, the rms values of pressure seem to be overestimated significantly outside the boundary layer and also in the whole boundary layer profile down to the wall, especially in case $A$. This result is consistent with the streamwise evolution outside the boundary layer plotted in fig. 8. Inside the boundary layer, the spurious noise propagating downstream from the inlet in cases $A$ and $B$ is refracted towards the wall as a result of the mean velocity gradient associated to the boundary layer profile ([108]). This means that the boundary layer acts as an acoustic wave guide for the inlet spurious noise propagating in the downstream direction, which can result in severe overestimations of the rms values of wall pressure. On the contrary, no overestimation of the rms values of pressure can be seen in cases $C$ compared with the DNS values, which confirms the favourable properties of this inlet condition in terms of spurious noise generation.

It should be emphasized that the comparison of the WMLES pressure levels with the DNS values is far from trivial since the resolved field in WMLES does not include all the dynamically active scales of turbulence. A significant portion of the near-wall velocity fluctuations is not resolved in the framework of WMLES, as well as a minor portion of the fluctuations in the outer layer (fig. 7). Using the acoustic analogy of Lighthill [58], the velocity fluctuations can be seen as quadrupole acoustic sources, which implies that some of the sources are missing when the near-wall dynamics is partially modelled. If these acoustic sources where incoherent with one another, one would expect the rms values of pressure to be underestimated in WMLES compared with DNS. However, there may be some correlation (hence coherence) between the turbulent velocity fluctuations at two different wall distances within the boundary layer profile, so that it is not 


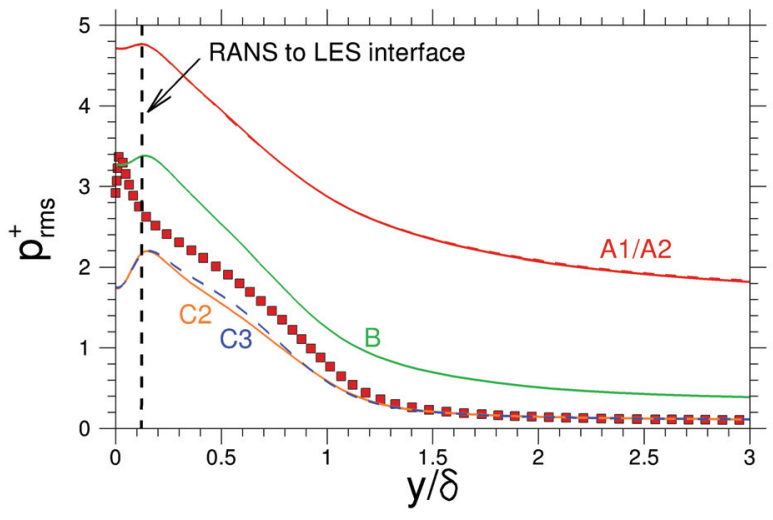

(a) Wall-normal distribution of pressure rms $P_{r m s}^{+}=\frac{P_{r m s}}{\rho u_{*}^{2}}$

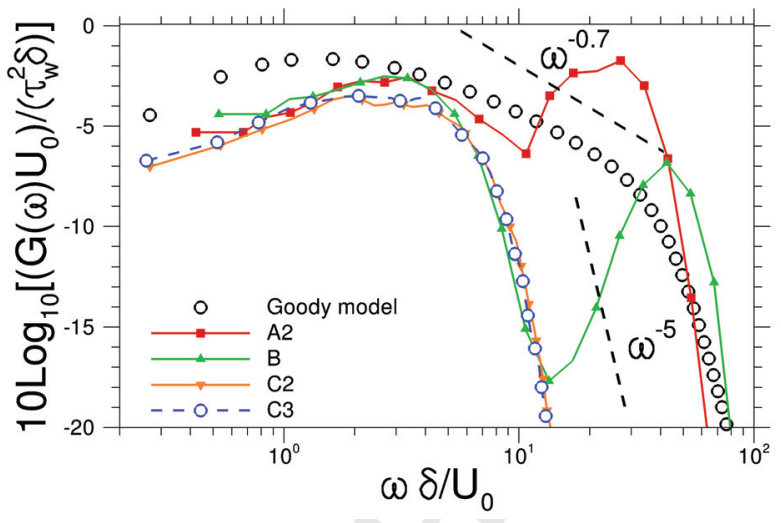

(b) PSD of wall pressure fluctuations

Figure 9: Wall pressure fluctuations at $R e_{\theta}=5200$

straightforward to predict whether an under- or overestimation should arise. A detailed discussion of the relationship between wall pressure and the turbulent velocity fluctuations including such non-intuitive cancellations of contributions of different regions may be found in Ref. [16].

In the context of WMLES, a relevant question is whether the turbulent fluctuations in the outer layer contribute significantly to the wall pressure signal, or at least to its low-frequency range. The flat-plate zero-pressure-gradient turbulent boundary layer considered here is fundamentally different, concerning its acoustic properties, from the streamwise-periodic channel flow which features two walls separated only by a distance of the same order of magnitude as the turbulent large scales. For this reason, the conclusions drawn in the pioneering work by Park and Moin [73] do not necessarily hold in the present case. One major reason for the differences in wall pressure fluctuations between channel flows and boundary layers is the different outer regions, for instance in terms of turbulent / non turbulent interface [98], as mentioned by Farabee and Casarella [37] who suggest that the major turbulent contributions to the low and high wave number ranges of the wall pressure spectrum may come from the outer and inner layers respectively. This suggestion is consistent with the contribution of the various parts of the boundary layer to the wall pressure spectrum quantitatively estimated by Aupoix [2], whose results may be used to conclude that if only the lower frequency range of the wall pressure spectrum is sought for a specific study of a vibration, dynamic loading or acoustics problem, then resolving only the outer layer by a WMLES may be sufficient at high Reynolds number. The range of the spectrum that may be resolved is related to the estimated fraction of wall pressure variance resolved by the WMLES. According to fig. 4 of Ref [2], if the upper half of the boundary layer profile (in logarithmic scale) is resolved (LES zone with negligible subgrid stresses) while the lower half is represented by the wall model (RANS zone), then at very high Reynolds numbers one may expect the resolved fraction of wall pressure variance to be approximately equal to one half. This is precisely what is expected if the RANS/LES interface is set at the geometric centre of the logarithmic layer $\left(d_{w}^{\text {interface, }+}=3.9 \sqrt{R_{\tau}}\right.$, see Ref. [80]). With the interface setting chosen for the present study $\left(d_{w}^{\text {interface }}=0.125 \delta\right.$ ), the expected high-Reynolds number trend is not as favourable, but this is not an issue at the present moderate Reynolds number. It should also be noted that an increasing importance of the outer layer towards higher Reynolds numbers for wall pressure is reported by Bernardini and Pirozzoli [7] in supersonic turbulent boundary layers as well. 
The one-sided Power Spectral Density (PSD) of wall pressure fluctuations named $G(f)$ (expressed in $P a^{2} / H z$ ) describes how the mean squared value of the wall pressure $P_{r m s}^{2}$ previously described is distributed in frequency since:

$$
P_{r m s}^{2}=\int_{0}^{\infty} G(f) d f=\int_{0}^{\infty} G(\omega) d \omega \quad \text { with } \quad \omega=2 \pi f
$$

(note that $G(f)$ and $G(\omega)$ differ by a factor of $2 \pi$ ). In the frame of wall-turbulence, the semi-empirical model proposed by Goody[45] satisfies the experimentally observed decay law $\omega^{-5}$ for high frequencies. What is more, this model takes account of the Reynolds number dependence and reads as:

$$
\frac{G(\omega) U_{0}}{\tau_{w}^{2} \delta}=\frac{C_{2}\left(\frac{\omega \delta}{U_{0}}\right)^{2}}{\left[\left(\frac{\omega \delta}{U_{0}}\right)^{0.75}+C_{1}\right]^{3.7}+\left[C_{3} R_{T}^{-0.57}\left(\frac{\omega \delta}{U_{0}}\right)\right]^{7}}
$$

where $C_{1}=0.5, C_{2}=3$ and $C_{3}=1.1$ are empirical constants. $R_{T}=\left(\delta / U_{0}\right) /\left(\nu / u_{\tau}^{2}\right)=R e_{\tau} \sqrt{C f / 2}$ is the ratio of the outer to inner time scale ranges of the wall signal, which represents the effect of the Reynolds number. Indeed, Goody[45] suggests $R_{T}=0.11 R e_{\theta}^{3 / 4}$ to yield best agreement with experimental data.

In the frame of WMLES simulations the single-point spectra for cases $A, B$ and $C$ are compared with Goody's model plotted in outer-scales in figure 9(b). The low frequency content of the spectra is somewhat underpredicted. As for very high frequencies, the spectra fall off too fast and do not follow the $\omega^{-5}$ decay law, which is not unexpected since the simulations are not DNS. This latter aspect is also observed in highly resolved WRLES [42]. Compared with calculation $C$, the excess in $P_{r m s}$ for calculations $A$ and $B$ (see figure $9(\mathrm{a})$ ) is due to the broadband peak observed at high frequencies $\left(\frac{\omega \delta}{U_{0}} \approx 25\right.$ for Case $A$ and $\frac{\omega \delta}{U_{0}} \approx 45$ for Case $\left.B\right)$. Shur et al[97] performed zonal RANS-IDDES on a flat plate boundary layer and observed similar spurious high frequency peaks with another synthetic turbulence method. In the frame of WMLES of channel flow calculations, Park and Moin[73] also observe spurious high wave number modes that contaminate the pressure spectra. In our case, the broadband peak observed for example in Case $A$ can be interpreted as the signature of spurious acoustics generated by the synthetic turbulence and propagated from the inlet. Indeed, the hydrodynamic field depicted in figure 6 is nearly the same for calculations $A$ and $C$. Moreover, some of the spurious spectral content in case $B$ is located at higher frequency than in case $A$. This can be explained by the fact that the white noise method, in which the fluctuations have little spatio-temporal correlation, does not involve scales as large as the SEM method does. In contrast, no excess can be seen in the spectra of cases $C$. This result shows again the drastic positive effect on the simulated pressure field brought by the proposed methodology illustrated by calculations $C$.

To get a deeper insight into the properties of the simulated pressure field, the Probability Density Functions (PDF) of the pressure fluctuations at $R e_{\theta}=5200$ are plotted in figure 10. The Gaussian distribution as well as the experimental results from Tsuji et al[113] are included. On linear scales (see figure 10(a)) the PDF of the different calculations are indistinguishable while on semi-logarithmic scales (see figure 10(b)) some deviations in the tails of the distribution are evidenced. Tsuji et al[113] showed that in the tail parts, the PDF shape deviates significantly from the Gaussian form and evidences the existence of rare high-amplitude wall-pressure fluctuations (see also the discussion by Schewe[90] and by Naka etal [68]). These authors have shown that this departure from Gaussian behaviour is observed in the outer region of the boundary layer as well, consistent with the findings by Farabee and Casarella[37] who identified the outerlayer intermittency as a source of wall-pressure fluctuations. Only the data from calculations $C$ show a good agreement with those of Tsuji et al [113]. Conversely, this important property of the wall pressure PDF is obtained neither with calculation $A$ nor with $B$ which both yield almost a Gaussian behaviour showing once again the long lasting impact 


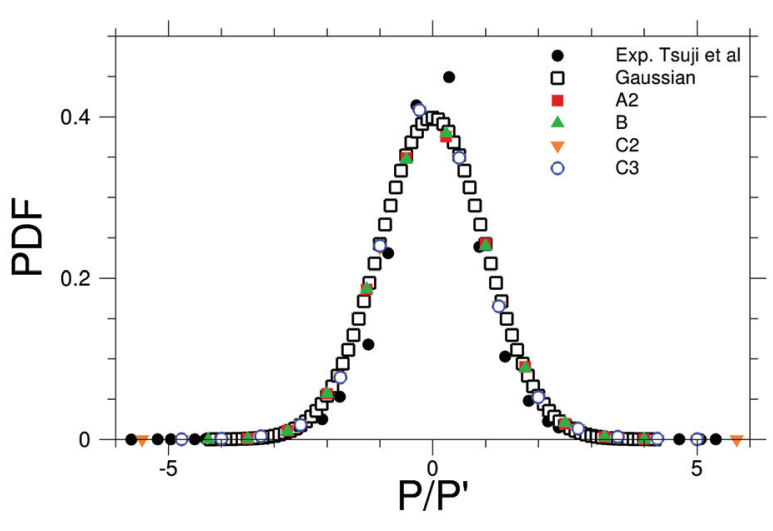

(a) linear scales

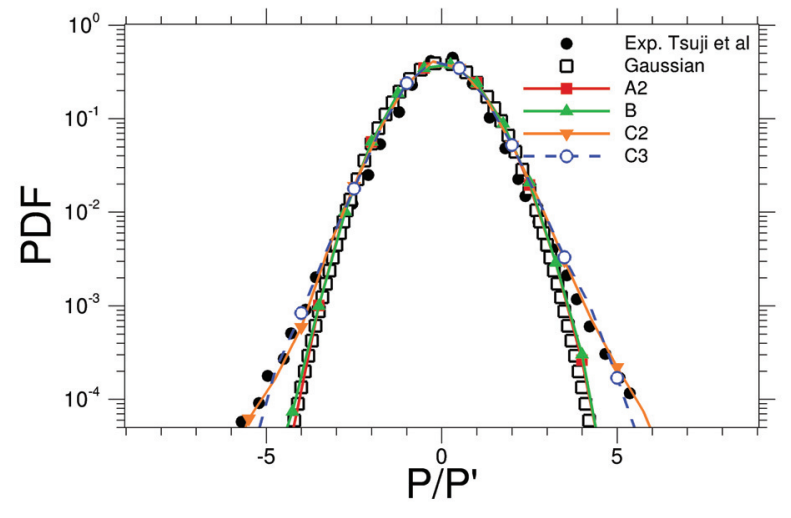

(b) semilogarithmic scales

Figure 10: PDF of wall pressure fluctuations normalized by their root mean squared value at $\operatorname{Re}_{\theta}=5200$.

of the inlet synthetic turbulence methods. This Gaussian behaviour may result from the random superimposition of spurious noise propagating from independent sources at the inlet, consistent with the central limit theorem. Note that the importance of the tripping conditions is also a major issue on the experimental side [61]. However, these results show also that the intermittent process concerning wall pressure can be simulated in a WMLES framework with the proposed method $C$ that is fully based on the use of source terms acting in the NS equations but not on the velocity field itself. Conversely, classical synthetic turbulence methods, though rather efficient to predict the velocity field, can completely corrupt the pressure field. From now on, the approach used in calculation $C_{3}$ is retained to investigate the flow dynamics around the three-element airfoil since the corresponding approach is efficient without contaminating the flow physics and especially the pressure field.

\section{Application on a three-element airfoil}

The three-element airfoil is a more complex geometry and a good outcome would be a positive indication for industrial application of the proposed methodology. The objective of this section is not to investigate the flow physics around a high-lift device (see [28] for a thorough investigation of the corresponding flow dynamics) but to assess the robustness that is needed from an advanced turbulence modelling method for "real-life" applications where both criteria I and II discussed in the introduction are essential. Indeed, the flow over a three-element airfoil is inherently complex and exhibits a wide range of physical phenomena including large low speed areas, strong pressure gradients, confluence of boundary layers and wakes as well as unsteadiness and three-dimensionality on fairly large scales. The existence of different flow regions induces conflicting demands on the grid and a host of technically challenging practical issues arise.

The proposed modelling methodology has been assessed on the LEISA three-element airfoil designed by DLR[123] for flow conditions corresponding to aircraft approach. The chord Reynolds number is $R e_{c}=\frac{U_{0} c}{\nu}=2.09 \times 10^{6}$ (with $U_{0}=51 \mathrm{~m} \cdot \mathrm{s}^{-1}$ and $c=0.6 \mathrm{~m}$ ), the planar $x-y$ grid has 380,000 points and the spanwise grid has $N_{z}=128$ points with $\Delta z / c=1.25 \times 10^{-3}$. The total number of grid points could be limited to less than $50 \times 10^{6}$ grid points thanks to the ZDES modelling. The time step is $\Delta t_{C F D} \cdot U_{0} / c=1.7 \times 10^{-9}$ i.e. $\Delta t^{+}=\frac{u_{*}^{2} \Delta t_{C F D}}{\nu}=0.1$ in the domain where mode 3 of ZDES is retained. The zonal strategy retained and the modes used for this simulation are depicted in the upper part of 
figure 11. One can notice that all modes of ZDES are used simultaneously in the same calculation since mode 1 is retained in the slat and flap coves (problem of category I, see table 2) while mode 2 is used on the upper side of the flap where the location of separation is not known a priori. The turbulent content of the boundary layer is rebuilt close to the main wing trailing edge thanks to mode 3 of ZDES. It is worth remembering that the computation of the wake of the main wing in LES mode including the evolution of wall turbulence over the main wing would require extremely fine grids beyond affordability. As an example, Terracol and Manoha [111] performed a WRLES on a similar three-element airfoil but on a twice smaller model $($ i.e. $c=0.3 \mathrm{~m}$ ) than the one used in this study. Their calculation is based on a 2.6 billion-point grid which required a large amount of CPU resources, namely 6 million CPU hours. This resolution far exceeds what would be feasible for an entire wing today. Note that taking into account the Reynolds number (i.e. chord length difference) there are more than two orders of magnitude on the total number of grid points between the WRLES grid by Terracol and Manoha [111] and the present ZDES grid [28]. Besides, the meshing strategy for ZDES is very simple since it uses only one mesh (one may simply refine a RANS grid) as opposed to more complex WMLES strategies involving for instance an embedded mesh needed for the wall model $([74,10])$ or an overlapping mesh strategy in the area of synthetic turbulence generation as Shur et al [97] do. Moreover, the propagation of acoustic waves through the turbulence injection domain is unaltered. This implies that acoustic feedback mechanisms are preserved, which is not as clear for other methods, e.g. resorting to internal damping layers in overlapping regions.

Two computational set-ups are considered. The first one is based on a combination of anisotropic white noise and dynamic forcing similar to Case $B$ for the flat plate boundary layer study (see §3.2) and will serve as reference calculation. The second set-up relies on the new approach based on the introduction of roughness elements with the ZIBC approach. Analogous to configuration $C_{3}$, an array of cylindrical roughness elements characterized by the height of the cylinder $h / \delta_{0}=0.3$ and the spanwise spacing $\lambda_{z} / \delta_{0}=0.5$ is considered. A zoom of the computational domain is depicted in figure 11(b) together with the distance to the wall $d_{w}$. The distance to the wall, required for both RANS and ZDES simulations based on the Spalart-Allmaras model, has been computed from the immersed boundary interface of the cylindrical roughness elements and from the classical no-slip conditions on the wing surface as discussed by Mochel et al [64]. It should be noted that the non-dimensionalised properties of the white noise or of the roughness elements are directly taken from the flat-plate test case without a case-specific optimisation in order to demonstrate the generality of the method. The streamwise (respectively spanwise) grid resolution is $\Delta x / \delta_{0}=0.086$ (respectively $\Delta z / \delta_{0}=0.026$ ) corresponding to $\Delta x^{+}=250$ (respectively $\Delta z^{+}=75$ ) when expressed in inner scales. The computational domain length where mode 3 is retained (see figure 11(a)) is $L_{x} / \delta_{0}=3.2$ making the WMLES very challenging. This permits to assess the efficiency of the proposed methodology. It should be noted that resolved turbulence is injected within the flow and not at a boundary of the numerical domain, which would not be feasible with the SEM boundary condition used in the flat-plate test case.

The salient features of the instantaneous flow field are highlighted in figure 12. The flow in the slat cove displays a large recirculation bubble bounded by a shear layer emanating from the slat cusp and reattaching near the slat trailing edge. Similarly, the flap cove also behaves like a shallow cavity. A higher value of the Q-criterion $Q \frac{c^{2}}{U_{0}^{2}}=3000$ allows one to make a zoom of the flow at the rear part of the main wing and its downstream evolution over the flap. Despite the very challenging grid and flow conditions, the LES content in the outer part of the boundary layer is quickly generated.

In figure 13, showing the instantaneous $-\frac{1}{\rho} \frac{\partial \rho}{\partial t}$ field, both sound waves and turbulent flow regions are evidenced. The contamination from the white noise application is clearly visible since nearly spherical waves can be seen from the region 


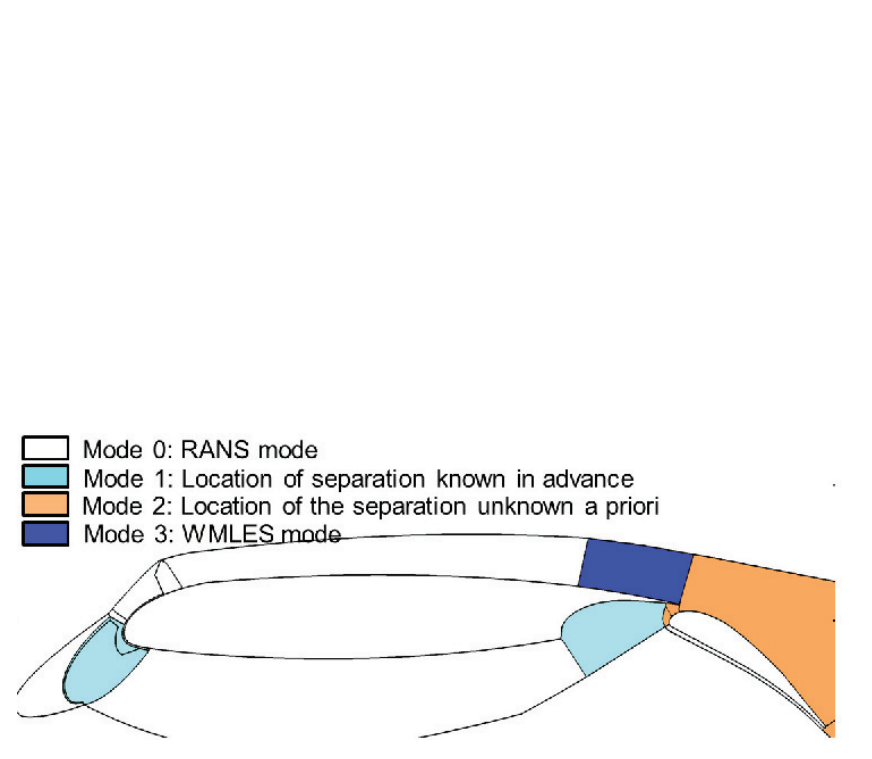

(a) ZDES zones for the calculation of the three-element airfoil

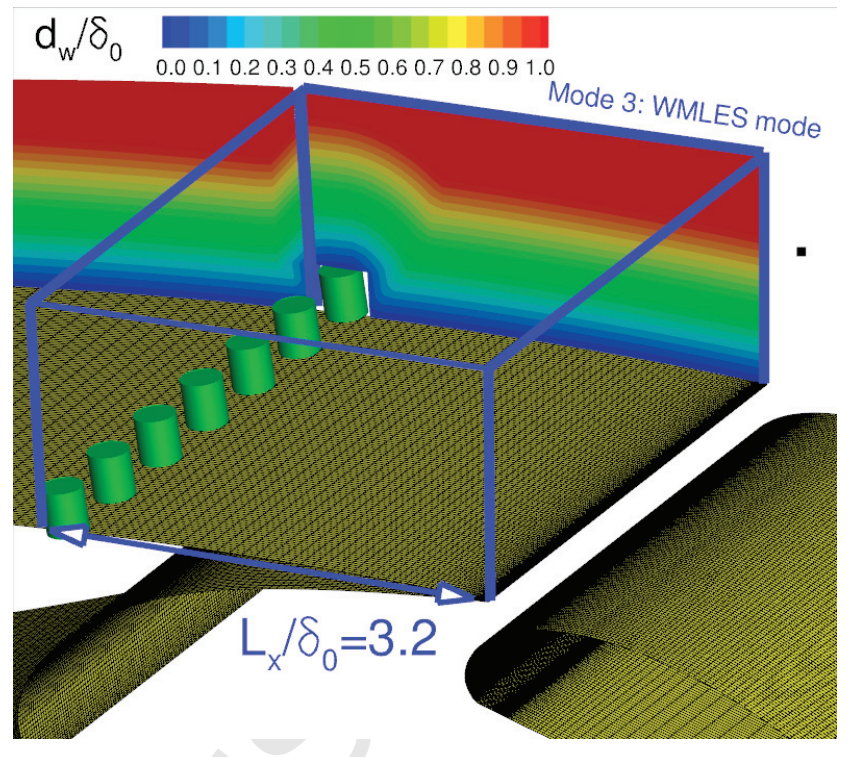

(b) Roughness elements in the WMLES domain similar to Case $C_{3}$ (see table 5).

Figure 11: Computational description. $d_{w} / \delta_{0}$ is the normalized distance to the wall where $\delta_{0}$ is the boundary thickness at the inlet domain.

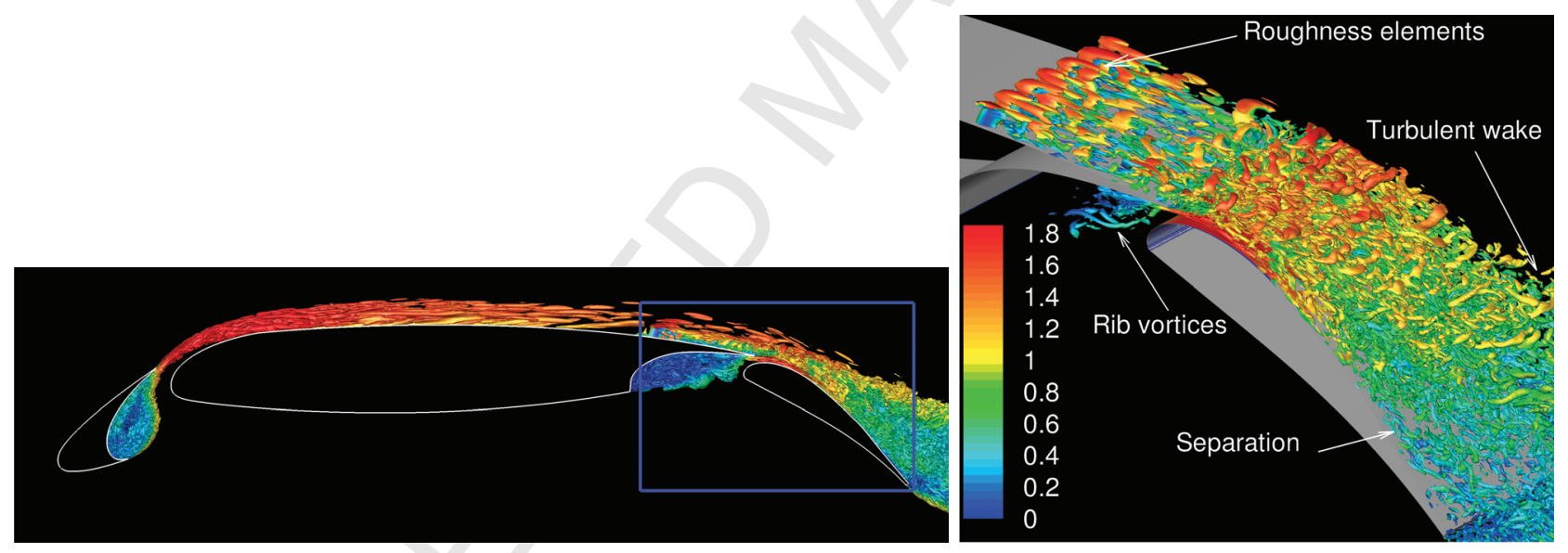

Figure 12: Isosurface of the $Q$ criterion coloured by the velocity magnitude $\|\mathbf{u}\| / U_{0}$ in the flap area (top view: $Q \frac{c^{2}}{U_{0}^{2}}=100 ;$ zoom: $Q \frac{c^{2}}{U_{0}^{2}}=3000$ ).

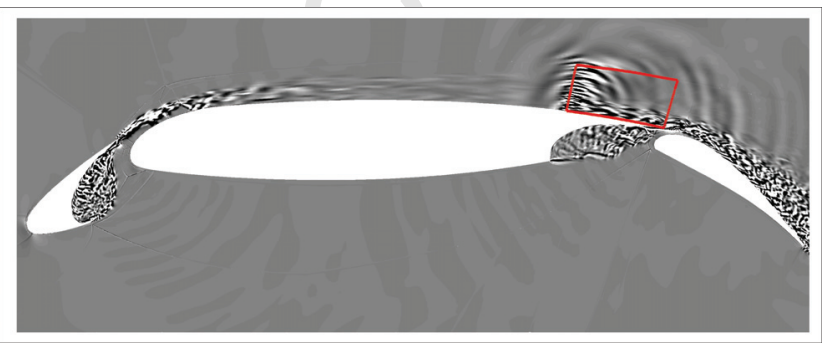

(a) ZDES mode 3 - anisotropic White Noise - Dynamic Forcing (similar to Case $B$ )

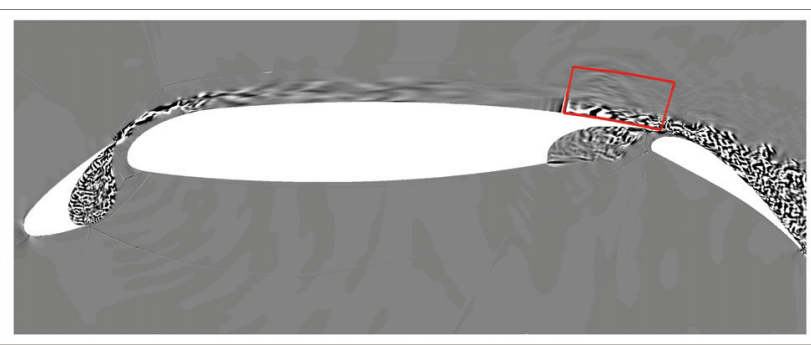

(b) ZDES mode 3 - ZIBC - Dynamic Forcing (similar to Case $\left.C_{3}\right)$

Figure 13: Instantaneous field of $-\frac{1}{\rho} \frac{\partial \rho}{\partial t}$. 


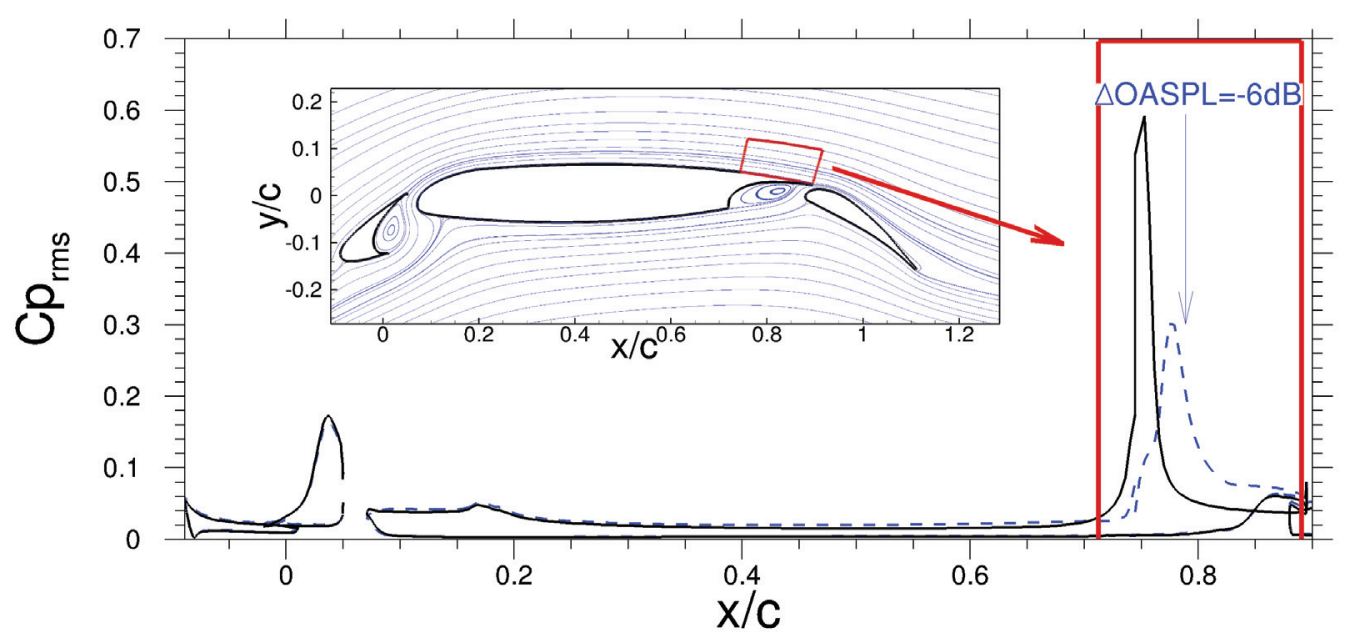

Figure 14: Root-mean squared pressure fluctuation along the wing.

very near the RANS (mode 0 domain) to WMLES (mode 3 domain) transition (see figure 13(a)). These waves appear to have their highest amplitude near the inlet boundary but contaminate the whole pressure field as discussed in [28]. Conversely, the calculation based on the immersed roughness elements induces significantly less spurious noise at the inlet (see figure 13(b)) since the use of non-moving obstacles has the great advantage of being steady and produces less spurious noise than the inflow method based on the introduction of random velocity fluctuations. This result is confirmed by figure 14 indicating a twice as low level of r.m.s. wall pressure fluctuations at the inlet. The higher values of $C p_{r m s}$ further downstream for the Case $C 3$ calculation is due to a different hydrodynamic behaviour of the flow rather than an aeroacoustic signature. This may probably be improved by setting the turbulent injection further upstream in order to reduce the possible interactions between the generation of resolved turbulence and the flow dynamics at the trailing edge (especially the fluctuations of circulation). On the contrary, the very short mode 3 domain retained for the present test case was devised to assess the robustness of the method for industrial applications.

\section{Conclusion}

A strategy for a rapid and low noise switch from an upstream RANS treatment to a downstream Wall-Modelled LES of attached boundary layers on curvilinear grids within compressible flow solvers has been presented. This turbulent inflow method can be embedded within the computational domain or used at the numerical inlet. It is devised for practical applications with WMLES grids around three-dimensional geometries and takes advantage of the flexibility provided by the zonal hybrid RANS/LES modelling. It relies on a physics-motivated combination of Zonal Detached Eddy Simulation as the WMLES technique together with a Dynamic Forcing method which processes the streamwise vorticity fluctuations created by a Zonal Immersed Boundary Condition describing roughness elements whose characteristics are inspired by the literature on optimal transient growth of perturbations of the turbulent boundary layer. This approach has favourable features in terms of turbulence quality, relaxation length, extra cost, complexity of implementation and initialisation of the calculation. Another crucial criterion is the low spurious noise generated by the present turbulent inflow. It is especially confirmed that the Dynamic Forcing technique has a limited acoustic footprint.

The new approach has been compared with other turbulent inflow methods relying on the Synthetic Eddy Method and 
on the injection of White Noise, on two test cases. The first case is a low Mach-number flat-plate zero-pressure-gradient turbulent boundary layer over a rather wide range of Reynolds numbers $3040 \leq R e_{\theta} \leq 6100$. The synthetic turbulence methods (SEM and White Noise) generate a strong spurious noise which dominates the physical noise generated by the turbulent boundary layer itself. On the contrary, the new approach has a low acoustic footprint thanks to the steady nature of the roughness elements used to trigger the initial fluctuations and because source terms in the momentum equations are involved rather than a direct manipulation of the velocities. Besides, the performance in generating a physically-sound turbulent flow field is equivalent to more common inflow methods. The generation of large-scale streamwise vorticity by the roughness elements is instrumental in recovering quickly a self-sustained resolved turbulence with the proper spatiotemporal correlation. The new approach is able to recover a mean skin friction within a $5 \%$ tolerance margin about the Coles-Fernholz correlation as soon as the forcing area is ending, i.e. after a relaxation distance close to only seven times the inlet boundary layer thickness. The pressure fields present the expected behaviour of turbulent flows as resolved by a WMLES method. A spectral analysis of the wall pressure signal confirms that the classical inflow methods induce a strong spurious noise (especially in the high-frequency range) whereas no footprint of the inflow condition can be seen in the spectrum associated to the new approach. Because the calculations are WMLES and not DNS, the highest frequencies of the spectrum are nevertheless not resolved. A deficit of energy at low frequency has been reported as well. Interestingly enough, only calculations with the new inflow condition predict properly the intermittent nature of the wall pressure signal observed experimentally, whereas more classical turbulent inlets induce a dominating almost-Gaussian spurious noise. This shows that the intermittent process concerning wall pressure can be simulated in a WMLES framework with the proposed method whereas classical synthetic turbulence methods, though rather efficient to predict the velocity field, can completely corrupt the pressure field. If this latter aspect is not crucial for the study, it should however be noted that the simple injection of anisotropic White Noise coupled with its processing by the Dynamic Forcing is able to regenerate self-sustainable resolved turbulence in the outer layer with much flexibility of use. One final note is that the estimation of the Probability Density Function of wall pressure seems to be a simple way of determining whether the pressure field resolved by the simulation is significantly polluted by spurious noise or not.

The second test case is the flow around a three-element airfoil representative of industrial applications. The robustness of the proposed approach is assessed by resorting to WMLES (ZDES mode 3) in the upper boundary layer near the trailing edge of the main airfoil element, whereas the other two modes of ZDES are used in other regions of the flow. Turbulence is injected only 3.2 boundary layer thicknesses upstream from the trailing edge. In spite of this challenging set-up, a successful generation of a resolved turbulent content in the outer layer near the trailing edge is observed, providing the proper wake interacting with the flap behind. The new approach generates significantly less spurious noise than the approach involving white noise. A reduction of $6 \mathrm{~dB}$ has been reported for the maximum OASPL on the upper surface of the main wing. Even better results could be expected if the turbulent injection were set further upstream than in this case meant for robustness assessment. Moreover, using ZDES implies a grid count saving of more than two orders magnitude in comparison with a Wall-Resolved LES and allows a very simple meshing strategy with only one mesh needed (no overlapping of multiple meshes contrary to some other methods for WMLES or for 'silent' turbulent injection).

This study has demonstrated the possibility to treat locally in WMLES a region of interest of the flow in a flexible hybrid RANS/LES framework and to alleviate the dominant spurious noise generation usually caused by classical turbulence injection methods. This kind of 'silent' WMLES may be of particular interest for aeroacoustics studies such as trailing edge noise prediction or jet noise prediction when the turbulent fluctuations from the attached boundary layers at the 
nozzle exit should be taken into account. There is also an incentive towards the prediction of turbulent boundary layer noise, for which the WMLES strategy may be used with special care to recognise the difference with a DNS. The discussion based on the available literature has shown that one may expect to resolve approximately $50 \%$ of the wall pressure variance by a WMLES at high Reynolds number if the turbulent fluctuations are resolved by the WMLES (rather than modelled) above the geometric centre of the logarithmic layer. The resolved fraction of the wall pressure variance corresponds approximately to the lower frequency part of the spectrum, which suggests that WMLES is a viable strategy to predict the low-frequency wall pressure fluctuations, if the description of the higher frequency range is not required or can be provided by an empirical model. A general discussion of the possibility to resort to partially resolved simulations for the prediction of aeroacoustic source terms may be found in Ref. [119]. The frequency range of interest can be low, for instance because of the vibrational properties of the skin and structure of the flight vehicle considered ([6]). The turbulent boundary layers along the fuselage of an aircraft are also a significant source of cabin noise, as indicated for instance by in-flight measurements recently performed in an Airbus A320 [11, 69]. The frequency range of interest depends on the transfer function associated with the fuselage skin and on the human perception of noise. The study of wall-pressure fluctuations in turbulent boundary layers, especially in presence of mean pressure gradients, is highly topical ([85, 88, 43]) and its prediction by means of computational fluid dynamics is not an easy question ([49]), although there is a need for a universal CFD strategy for wall pressure prediction in the context of aircraft interior noise prediction (see Ref. [48] where it is emphasized that resolving only the largest scales of wall turbulence may be sufficient due to poor transmission efficiency of the higher frequencies). Because of the broad scale separation at the Reynolds numbers of applications, wall-modelling is needed to keep the computational cost of aeroacoustic studies within reach, making the use of hybrid RANS/LES techniques essential ([57]).

\section{Acknowledgments}

The authors wish to thank all the people involved in the past and present evolution of the FLU3M code. The ZIBC approach has been developed in the framework of the research project ALLIGATOR funded by ONERA. Part of this work is also related to the EU collaborative research project Go4Hybrid, funded by the European Community in the 7th Framework Programme, under Contract No. 605361.

\section{References}

[1] Araya, G., Castillo, L., Meneveau, C., Jansen, K., 2011. A dynamic multi-scale approach for turbulent inflow boundary conditions in spatially developing flows. Journal of Fluid Mechanics, vol.670, pp 581-605.

[2] Aupoix, B., 2015. Extension of Lysak's Approach to Evaluate the Wall Pressure Spectrum for Boundary Layer Flows. Flow Turbulence and Combustion, vol 94, No. 1, pp 63-78.

[3] Aupoix, B., Arnal, D., Bezard, H., Chaouat, B., Chedebergne, F., Deck, S., Gleize, V., Grenard, P., Laroche, E., 2011. Transition and Turbulence Modeling. Aerospace Lab Journal, vol 2., AL02-01.

[4] Batten, P., Goldberg, U., Chakravarthy, S., 2004. Interfacing Statistical Turbulence Closures with Large-EddySimulation. AIAA J., vol.42, No.3, pp 485-492, March. 
[5] Beresh, S., Henfling, J., Spillers, R., Pruett, O., 2011. Fluctuating wall pressures measured beneath a supersonic turbulent boundary layer. Physics of Fluids, vol 23, 075110.

[6] Beresh, S. J., Henfling, J. F., Spillers, R. W., Pruett, B. O. M., 2013. Very-large-scale coherent structures in the wall pressure field beneath a supersonic turbulent boundary layer. Physics of Fluids 25, 095104/1-19.

[7] Bernardini, M., Pirozzoli, S., 2011. Wall pressure fluctuations beneath supersonic turbulent boundary layers. Physics of Fluids, 23, 085102 .

[8] Bies, D., Hansen, C., 2009. Engineering noise control. Theory and practice. Fourth edition. Spon Pres.

[9] Bocquet, S., Jouhaud, J.-C., Deniau, H., Boussuge, J.-F., Esteve, M., 2014. Wall-Modelled-Large-Eddy Simulation of a hot Jet-In-Cross-Flow with turbulent inflow generation. Computer \& Fluids, vol 101, pp 136-154.

[10] Bodart, J., Larsson, J., Moin, P., 2013. Large eddy simulation of high-lift devices. In: 21st AIAA Computational Fluid Dynamics Conference. No. AIAA 2013-2724.

[11] Bodén, H., G.Efraimsson, 2013. Aeroacoustics research in Europe: The CEAS-ASC report on 2012 highlights. Journal of Sound and Vibration 332, 6617-6636.

[12] Bradshaw, P., Feriss, D., Atwell, N., 1967. Calculation of boundary layer development using the turbulent kinetic energy equation. Journal of Fluid Mechanics, vol.28, pp 593-616.

[13] Bühler, S., Obrist, D., Kleiser, L., 2014. Laminar and turbulent nozzle-jet flows and their acoustic near-field. Physics of Fluids 26, 086103-1/17.

[14] Cambier, L., Heib., S., Plot, S., 2013. The Onera elsA CFD software: input from research and feedback from industry. Mechanics and Industry, vol 14, No 3, pp 159-174, doi:10.1051/meca/2013056.

[15] Chalot, F., Dagrau, F., Mallet, M., Normand, P., Yser, P., 2015. DIHOM: Industrialization of High-Order Methods - A Top-Down Approach. Vol. 128 of NNFM. N. Kroll et al., Ch. Higher-Order RANS and DES in an Industrial Stabilized Finite Element Code, pp. 489-519.

[16] Chang III, P. A., Piomelli, U., Blake, W. K., 1999. Relationship between wall pressure and velocity-field sources. Physics of Fluids 11, 3434-3448.

[17] Chauvet, N., Deck, S., Jacquin, L., 2007. Zonal-Detached-Eddy Simulation of a Controlled Propulsive Jet. AIAA J., vol 45, No. 10, pp 2458-2473.

[18] Choi, H., Moin, P., 1994. Effect of the computational time step on the numerical solution of turbulent flows. Journal of Computational Physics, vol. 113, no 1, pp 227-234.

[19] Choi, H., Moin, P., 2012. Grid-point requirements for large eddy simulation: Chapman's estimates revisited. Physics of Fluids, vol 24, 011702.

[20] Choi, J.-I., Edwards, J., Baurle, R., 2009. Compressible Boundary-Layer Predictions at High Reynolds Number Using Hybrid LES/RANS methods. AIAA Journal, vol 47, No. 9, pp 2179-2193. 
[21] Colonius, T., Lele, S., 2004. Computational aeroacoustics: progress on non-linear problems of sound generation. Progress in Aerospace Sciences, vol.40, pp 345-416.

[22] Cossu, C., Pujals, G., Depardon, S., 2009. Optimal transient growth and very large-scale structures in turbulent boundary layers. Journal of Fluid Mechanics, vol.619, pp 79-94.

[23] De la Puente, F., Sanders, L., Vuillot, F., Manoha, E., 2015. Nose landing gear flow and noise predictions on unstructured grid using a cell-centered Navier-Stokes code. 21th AIAA/CEAS AeroAcoustics Conference, Dallas, Texas USA, AIAA Paper 2015-3256.

[24] de la Puente Cerezo, F., Sanders, L., Vuillot, F., Druault, P., Manoha, E., 2017. Zonal Detached Eddy Simulation of a simplified nose landing-gear for flow and noise predictions using an unstructured Navier-Stokes solver. Journal of Sound and Vibration 405, 86-111.

[25] Deck, S., 2005. Zonal-Detached Eddy Simulation of the flow around a high-lift configuration. AIAA Journal, vol 43. , No. 11, pp 2372-2384.

[26] Deck, S., 2012. Recent improvements of the Zonal Detached Eddy Simulation (ZDES) formulation. Theoretical and Computational Fluid Dynamics, 26 (6), pp 523-550, doi: 10.1007/s00162-011-0240-z.

[27] Deck, S., Gand, F., Brunet, V., Ben Khelil, S., 2014. High-fidelity simulations of unsteady civil aircraft aerodynamics: stakes and perspectives. application of zonal detached eddy simulation. Phil. Trans. R. Soc. A., 372: 20130325, (doi: 10.1098/rsta.2013.0325).

[28] Deck, S., Laraufie, R., 2013. doi: 10.1017/jfm.2013.363. Numerical investigation of the flow dynamics past a threeelement aerofoil. Journal of Fluid Mechanics 732, 401-444.

[29] Deck, S., Renard, N., Laraufie, R., Sagaut, P., 2014, doi: 10.1063/1.4866180. Zonal Detached Eddy Simulation (ZDES) of a spatially developing flat plate turbulent boundary layer over the Reynolds number range $3150<R e_{\theta}<$ 14000. Physics of Fluids 26 (2), 025116.

[30] Deck, S., Renard, N., Laraufie, R., Weiss, P., 2014. Large scale contribution to mean wall shear stress in high Reynolds number flat plate boundary layers up to $\mathrm{R}_{\theta}=13650$. Journal of Fluid Mechanics 743, 202-248.

[31] Deck, S., Weiss, P., Pamiès, M., Garnier, E., 2008. On the Use of Stimulated Detached Eddy Simulation (SDES) for Spatially Developing Boundary Layers. Advances in Hybrid RANS-LES Modelling, NNFM97, pp 67-76, edited by S-H Peng and W. Haase, Springer-Verlag, BerlinHeidelberg .

[32] Deck, S., Weiss, P., Pamiès, M., Garnier, E., 2011. Zonal Detached Eddy Simulation of a spatially developing flat plate turbulent boundary layer. Computer \& Fluids, vol 48, pp 1-15, doi:10.1016/j.compfluid.2011.03.09.

[33] DeGraaff, D., Eaton, J., 2000. Reynolds number scaling of the flat-plate turbulent boundary layer. Journal of Fluid Mechanics, vol 422, pp 319-346.

[34] di Mare, L., Klein, M., Jones, W., Janicka, J., 2006. Synthetic turbulence inflow conditions for large-eddy simulation. Physics of Fluids, vol 18, 025107. 
[35] Duriez, T., Aider, J.-L., Wesfreid, J., 2009. Self-Sustaining Process through Streak Generation in a Flat-Plate Boundary Layer. Physical Review Letters, Vol.103, No. 14, 144502.

[36] Fadlun, E. A., Verzicco, R., Orlandi, P., Mohd-Yusof, J., 2000. Combined Immersed-Boundary/Finite-Difference Methods for Three-Dimensional Complex Flow Simulations. Journal of Computational Physics 161 (1), 35-60.

[37] Farabee, T., Casarella, M., 1991. Spectral features of wall pressure fluctuations beneath turbulent boundary layers. Phys. Fluid. A, 3(10), pp 2410-2420.

[38] Fukagata, K., Iwamoto, K., Kasagi, N., 2002. Contribution of reynolds stress distribution to the skin friction in wall-bounded flows. Physics of Fluids, 14, L73.

[39] Gand, F., 2016. Investigation of turbulence development in incompressible jets with Zonal Detached Eddy Simulation (ZDES) and synthetic turbulent inflow. International Journal of Heat and Fluid Flow, vol 61, pp 425-437.

[40] Garnier, E., Adams, N., Sagaut, P., 2009. Large-Eddy Simulation for Compressible Flows. Springer-Verlag, Berlin.

[41] Glatzer, C., MeiB, J.-H., Meinke, M., Schroeder, W., 2011. Numerical investigation of the near wake of generic space launcher systems at transonic and supersonic flows. In: 4th European Conference For Aerospace Science, Saint Petersburg, Russia.

[42] Gloerfelt, X., Berland, J., 2013. Turbulent boundary layer noise: direct radiation at mach number 0.5. Journal of Fluid Mechanics 723, 318-351.

[43] Gloerfelt, X., Cohen, E., 2016. Influence of pressure gradients and Reynolds number on wall-pressure wavenumberfrequency spectra. In: 22nd AIAA/CEAS Aeroacoustics Conference. No. AIAA 2016-2910.

[44] Gloerfelt, X., Robinet, J., 2013. A silent condition for the study of boundary layer noise. 19th AIAA/CEAS AeroAcoustics Conference, Berlin, Germany, AIAA Paper 2013-2247.

[45] Goody, M., 2004. Empirical Spectral Model of Surface Pressure Fluctuations. AIAA J. vol.42, No.9, pp 1788-1794.

[46] Han, Y., Ding, G., He, Y., Wu, J., Le, J., 2018. Assessment of the IDDES method acting as wall-modeled LES in the simulation of spatially developing supersonic flat plate boundary layers. Engineering Applications Of Computational Fluid Mechanics 12 (1), 89-103.

[47] Jarrin, N., Benhamadouche, S., Laurence, D., Prosser, R., 2006. A Synthetic-Eddy-Method for generating inflow conditions for Large Eddy Simulation. International Journal of Heat and Fluid Flows, Vol.27, pp 585-593.

[48] Jiricek, O., 2016. Aeroacoustics research in Europe: The CEAS-ASC report on 2015 highlights. Journal of Sound and Vibration 381, 101-120.

[49] Juvé, D., Berton, M., Salze, E., 2015. Spectral properties of wall-pressure fluctuations and their estimation from Computational Fluid Dynamics. In: Ciappi, E., Rosa, S. D., Franco, F., Guyader, J.-L., Hambric, S. A. (Eds.), Flinovia - Flow Induced Noise and Vibration Issues and Aspects. Springer.

[50] Keating, A., De Prisco, G., Piomelli, U., 2006. Interface conditions for hybrid RANS/LES calculations. International Journal of Heat and Fluid Flows, 27: 777-788. 
[51] Keating, A., Piomelli, U., 2006. A dynamic stochastic forcing method as a wall-layer model for large-eddy simulation. Journal of Turbulence, vol.7, (12), pp 1-24.

[52] Keating, A., Piomelli, U., Balaras, E., Kaltenbach, H., 2004. a priori and a posteriori tests of inflow conditions for large-eddy simulation. Physics of Fluids, vol.16, No. 12, pp 4696-4712.

[53] Klein, M., Sadiki, A., Janicka, J., 2003. A digital filter based generation of inflow data for spatially developing direct numerical simulation or large eddy simulation. Journal of Computational Physics, 186, pp 653-665.

[54] Laraufie, R., Deck, S., 2013. Assessment of Reynolds stresses tensor reconstruction methods for synthetic turbulent inflow conditions. Application to hybrid RANS/LES methods. International Journal of Heat and Fluid Flow, vol. 42, pp 68-78.

[55] Laraufie, R., Deck, S., Sagaut, P., 2011. A dynamic forcing method for unsteady turbulent inflow conditions. Journal of Computational Physics, vol. 230, pp 8647-8663, doi 10.1016/j.jcp.2011.08.012.

[56] Laraufie, R., Deck, S., Sagaut, P., 2011. A rapid switch from RANS to WMLES for spatially developping boundary layers. In: Ed. S. Fu, W. Haase, S. P., Schwamborn, D. (Eds.), Progress in Hybrid RANS-LES modeling. Notes on Numerical Fluid Mechanics and Multidisciplinary Design vol 117, pp 147-156. Springer.

[57] Lele, S. K., Nichols, J. W., 2014. A second golden age of aeroacoustics? Philosophical Transactions of the Royal Society A 372, 20130321/1-18.

[58] Lighthill, M. J., 1952. On sound generated aerodynamically. I. General theory. Proceedings of the Royal Society A $221,564-587$.

[59] Liou, M., 1996. A sequel to AUSM: AUSM+. Journal of Computational Physics, vol. 126, pp 364-382.

[60] Lund, T., Wu, X., Squires, K., 1998. Generation of Turbulent Inflow Data for Spatially-developping Boundary Layer Simulations. Journal of Computational Physics, vol.140, pp 233-258.

[61] Marusic, I., Chauhan, K., Kulandaivelu, V., Hutchins, N., 2015. Evolution of zero-pressure-gradient boundary layers from different tripping conditions. Journal of Fluid Mechanics 783, 379-411.

[62] Mary, I., Sagaut, P., 2002. Large eddy simulation of flow around an airfoil near stall. AIAA J., vol.40, No.6, pp $1139-1145$.

[63] Mittal, R., Iaccarino, G., 2005. Immersed boundary methods. Annual Review of Fluid Mechanics 37, $239-261$.

[64] Mochel, L., Weiss, P., Deck, S., 2014. Zonal Immersed Boundary Conditions: Application to a high Reynolds number afterbody flow. AIAA Journal, vol 52., No. 12, pp 2782-2794.

[65] Mohd-Yusof, J., 1997. Combined Immersed-Boundary/B-Spline Methods for Simulations of Flows in Complex Geometries. Annual Research Briefs, Center for Turbulence Research, 317-328.

[66] Morgan, B., Larsson, J., Kawai, S., Lele, S., 2011. Improving low-frequency characteristics of recycling/rescaling inflow turbulence generation. AIAA J., vol.49, pp 582-597. 
[67] Nagib, H., Chauhan, K., Monkewitz, P., 2007. Approach to an asymptotic state for zero pressure gradient turbulent boundary layers. Philosophical Transactions of the Royal Society A, 365, 755-770.

[68] Naka, Y., Stanislas, M., Foucaut, J.-M., Coudert, S., Laval, J.-P., Obi, S., 2015. Space-time pressure-velocity correlations in a turbulent boundary layer. Journal of Fluid Mechanics 771, 624-675.

[69] Norambuena, M., Böswald, M., Govers, Y., 2016. Vibro-acoustic analysis of flight test data comprising fuselage vibrations, external pressure and interior cabin noise measurements. In: 54th AIAA Aerospace Sciences Meeting. No. AIAA 2016-1013.

[70] Örlü, R., Schlatter, P., 2011. Inflow length and tripping effects in turbulent boundary layers. Journal of Physics: Conference series, vol.318, 022018.

[71] Österlund, J. M., Johansson, A. V., Nagib, H. M., Hites, M. H., 2000. A note on the overlap region in turbulent boundary layers. Physics of Fluids 12 (1), 1-4.

[72] Pamiès, M., Weiss, P., Garnier, E., Deck, S., Sagaut, P., 2009. Generation of synthetic turbulent inflow data for large eddy simulation of spatially evolving wall-bounded flows. Physics of Fluids, vol 21, 045103.

[73] Park, G., Moin, P., 2016. Space-time characteristics of wall-pressure and wall shear-stress flucuations in wall-modeled large eddy simulation. Physical Review Fluids, Vol. 1, 024404.

[74] Park, G. I., Moin, P., 2014. An improved dynamic non-equilibrium wall-model for large eddy simulation. Physics of Fluids 26, 015108.

[75] Péchier, M., Guillen, P., Caysac, R., 2001. Magnus Effect Over Finned Projectiles. AIAA Journal of Spacecraft and Rockets, vol.38, No.4, pp. 542-549.

[76] Piomelli, U., Balaras, E., 2002. Wall-layer models for Large-Eddy Simulations. Annual Review of Fluid Mechanics, $34: 348-74$.

[77] Poletto, R., Craft, T., Revell, A., 2013. A New Divergence Free Synthetic Eddy Method for the reproduction of inlet flow conditions for LES. Flow Turbulence and Combustion, vol 91, No. 3, pp 519-539.

[78] Powell, A., 1964. Theory of vortex sound. The Journal of the Acoustical Society of America 36 (1), $177-195$.

[79] Pujals, G., Depardon, S., Cossu, C., 2010. Drag reduction of a 3D bluff body using coherent streamwise streaks. Exp. Fluids, vol. 49, pp 1085-1094.

[80] Renard, N., Deck, S., 2015. Improvements in the formulation of Zonal Detached Eddy Simulation for Wall-Modeled Large-Eddy Simulation. AIAA Journal, vol 53., No. 11, pp 3499-3504.

[81] Renard, N., Deck, S., 2015. On the scale-dependent turbulent convection velocity in a spatially developing flat plate turbulent boundary layer at Reynolds number $\mathrm{Re}_{\theta}=13000$. Journal of Fluid Mechanics 775, 115-148.

[82] Renard, N., Deck, S., 2016. A theoretical decomposition of mean skin friction generation into physical phenomena across the boundary layer. Journal of Fluid Mechanics 790, 339-367. 
[83] Richez, F., Lepape, A., Costes, M., 2015. Zonal Detached Eddy Simulation of Separated Flow Around a Finite-Span Wing. AIAA Journal, vol 53., No 11, pp 3157-3166.

[84] Roidl, J., Meinke, M., Schroeder, W., 2011. Numerical investigation of shock wave boundary-layer interaction using a zonal rans-les ansatz. In: Ed. W.E. Nagel, D. K., Resch, M. (Eds.), High-Performance Computing in Science and Engineering'10.

[85] Rozenberg, Y., Robert, G., Moreau, S., 2012. Wall-pressure spectral model including the adverse pressure gradient effects. AIAA Journal 50 (10), 2168-2179.

[86] Sagaut, P., Deck, S., Terracol, M., 2013. Multiscale and multiresolution approaches in turbulence- LES, DES and hybrid RANS/LES methods: applications and guidelines (2nd Edition). Imperial College Press, London, UK, 448 pages.

[87] Sagaut, P., Garnier, E., Tromeur, E., Larchevêque, L., Labourasse, E., 2004. Turbulent inflow conditions for largeeddy simulation of compressible wall-bounded flows. AIAA Journal 42 (3), 469-477.

[88] Salze, E., Bailly, C., Marsden, O., Jondeau, E., Juvé, D., 2015. An experimental investigation of wall pressure fluctuations beneath pressure gradients. In: 21st AIAA/CEAS Aeroacoustics Conference. No. AIAA 2015-3148.

[89] Sandham, N., Yao, Y., Lawal, A., 2003. Large-eddy simulation of transonic turbulent flow over a bump. International Journal of Heat and Fluid Flows, Vol. 24, pp 584-595.

[90] Schewe, G., 1983. On the structure and resolution of wall-pressure fluctuations associated with turbulent boundarylayer flow. Journal of Fluid Mechanics, vol. 134, pp 311-328.

[91] Schlatter, P., Örlü, R., 2010. Assessment of direct simulation data of turbulent boundary layers. Journal of Fluid Mechanics, vol.659, pp 116-126.

[92] Schluter, J., Pitsch, H., Moin, P., 2004. Large Eddy simulation inflow conditions for coupling with Reynolds-averaged flow solvers. AIAA J., vol.42, No.3, pp 478-484.

[93] Sescu, A., Hixon, R., 2013. Toward low-noise synthetic turbulent inflow conditions for aeroacoustics calculation. International Journal for Numerical Methods in Fluids, vol.73, pp 1001-1010.

[94] Shen, M., Edwards, J., 2016. Development of a new RANS-LES Formulation. AIAA paper 2006-0898, 46th AIAA Fluid Dynmics Conference, Washington, DC, June.

[95] Shur, M., Spalart, P., Strelets, M., 2005. Noise prediction for increasingly complex jets. Part I: Methods and tests. International Journal of Aeroacoustics, 4: 247-256.

[96] Shur, M., Spalart, P., Strelets, M., Travin, A., 2008. A hybrid RANS-LES approach with delayed-DES and wallmodelled LES capabilities. International Journal of Heat and Fluid Flows, 29(6): 1638-1649.

[97] Shur, M., Spalart, P., Strelets, M., Travin, A., 2014. Synthetic Turbulence Generators for RANS-LES Interfaces in Zonal Simulations of Aerodynamic and Aeroacoustic Problems. Flow Turbulence and Combustion, vol 93, No 1, pp 63-92. 
[98] Sillero, J., Jimenez, J., Moser, R., 2013. One-point statistics for turbulent wall-bounded flows at Reynolds numbers up to $\delta^{+} \approx 2000$. Physics of Fluids 25, 105102 .

[99] Simon, F., Deck, S., Guillen, P., Caysac, R., Merlen, A., 2006. Zonal Detached Eddy Simulation of projectiles in the Subsonic and Transsonic Regimes. AIAA J.,vol. 45, No 7, pp 1606-1619.

[100] Simon, F., Deck, S., Guillen, P., Sagaut, P., Merlen, A., 2007. Numerical simulation of the compressible mixing layer past an axisymmetric trailing edge. Journal of Fluid Mechanics, vol 591, pp 215-253, Nov. 2007.

[101] Slotnick, J., Khidadoust, A., Alonso, J., Darmofal, D., Gropp, W., Lurie, E., Mavriplis, D., 2013. CFD Vision 2030 study: A path to revolutionary computational aerosciences. Tech. rep., NASA Langley Research Center, 23681-2199.

[102] Smirnov, A., Shi, S., Celik, I., 2001. Random flow generation technique for large eddy simulations and particledynamics modeling. Journal of Fluids Engineering, Vol. 123, pp 359-371, June.

[103] Spalart, P., Allmaras, S., 1994. A one equation turbulence model for aerodynamic flows. La Recherche Aérospatiale, vol 1., pp 5-21, Jan.

[104] Spalart, P., Jou, W., Strelets, M., Allmaras, S., 1998. Comments on the feasibility of LES for wings and on a hybrid RANS/LES approach. In Proceedings pp 137-147, 1st AFSOR Int. Conf. on DNS/LES, Ruston.

[105] Spalart, P., Strelets, M., Travin, A., 2006. Direct numerical simulation of large-eddy-break-up devices in a boundary layer. International Journal of Heat and Fluid Flows, 27(5), 903-910.

[106] Spille-Kohoff, A., Kaltenbach, H., 2001. Generation of turbulent inflow data with a described shear-stress profile. In Proceedings pp 137-147, third AFOSR Int. Conf. on DNS/LES, Arlington, edited by C.Liu, L. Sakell and T. Beutner, Greyden press, Columbus, OH, 5-9 August.

[107] Subbareddy, P., Bartkowicz, M., Candler, G., 2014. Direct numerical simulation of high-speed transition due to an isolated roughness element. Journal of Fluid Mechanics 748, 848-878.

[108] Suzuki, T., Lele, S. K., 2003. Green's functions for a source in a boundary layer: direct waves, channelled waves and diffracted waves. Journal of Fluid Mechanics 477, 129-173.

[109] Tabor, G., Baba-Ahmadi, M., 2010. Inlet conditions for large eddy simulation: a review. Computer \& Fluids, vol 39 , pp 553-567.

[110] Terracol, M., 2006. A zonal RANS/LES approach for noise sources prediction. Flow Turbulence and Combustion, vol 77, pp 161-184.

[111] Terracol, M., Manoha, E., 2014. Wall-resolved Large Eddy Simulation of a high-lift airfoil: detailed flow analysis and noise generation study. AIAA Paper 2014-3050.

[112] Terracol, M., Manoha, E., Murayama, M., Yamamoto, K., 2015. Aeroacoustic Calculations of the 30P30N High-lift Airfoil using Hybrid RANS/LES methods: Modeling and Grid Resolution Effects. 21th AIAA/CEAS AeroAcoustics Conference, Dallas, Texas USA, AIAA Paper 2015-3132. 
[113] Tsuji, Y., Fransson, J., Alfredsson, P., Johansson, A., 2007. Pressure statistics and their scaling in high-reynoldsnumber turbulent boundary layers. Journal of Fluid Mechanics, vol. 585, pp 1-40.

[114] Tucker, P., 2013. Unsteady computational fluid dynamics in aeronautics. Springer.

[115] Verriere, J., Gand, F., Deck, S., 2016. Zonal Detached Eddy Simulation of a dua-stream jet. AIAA Journal, vol 54., No. 10, pp 3176-3190.

[116] Viazzo, S., Dejoan, A., Schistel, R., 2001. Spectral features of the wall-pressure fluctuations in turbulent wall flows with and without perturbations using LES. International Journal of Heat and Fluid Flow, vol. 22, pp 39-52.

[117] Waindim, M., Gaitonde, D., 2016. A body-force method to generate supersonic equilibrium turbulent boundary layers profiles. Journal of Computational Physics, vol. 304, pp 1-16.

[118] Wang, M., Fattah, R. J., Angland, D., Zhang, X., 2015. High-order hybrid cell-centered method for computational aeroacoustics. In: 21st AIAA/CEAS Aeroacoustics Conference, AIAA AVIATION, Dallas, TX. No. AIAA 2015-3279.

[119] Wang, M., Freund, J. B., Lele, S. K., 2006. Computational prediction of flow-generated sound. Annual Review of Fluid Mechanics 38, 483-512.

[120] Weiss, P., Deck, S., 2011. Control of the antisymmetric mode $(\mathrm{m}=1)$ for high reynolds axisymmetric separating/reattachinf flows. Physics of Fluids, 23, 095102.

[121] Weiss, P., Deck, S., 2017. On the coupling of a zonal body-fitted/immersed boundary method with ZDES: application to the interactions on a realistic space launcher afterbody flow. Computers \& Fluids, in press, doi:10.1016/j.compfluid.2017.06.015.

[122] Wilcox, D., 2006. Turbulence modeling for CFD. DCW Industries.

[123] Wild, J., Pott-Pollenske, M., Nagel, B., 2006. An integrated design approach for low noise exposing high-lift devices. AIAA Paper 2006-2843.

[124] Winkler, J., Sandberg, R. D., Moreau, S., 2012. Direct numerical simulation of the self-noise radiated by an airfoil in a narrow stream. In: $18^{\text {th }}$ AIAA/CEAS Aeroacoustics Conference ( $33^{\text {rd }}$ AIAA Aeroacoustics Conference), June 2012, Colorado Springs, CO. No. AIAA 2012-2059.

[125] Wu, X., 2017. Inflow Turbulence Methods. Annual Review of Fluid Mechanics 49, 23-49.

[126] Xie, Z., Castro, I., 2008. Efficient Generation of Inflow Conditions for Large Eddy Simulation of Street-Scale Flows. Flow Turbulence and Combustion, vol 81, No. 3, pp 449-470.

[127] Ye, Q., Schrijer, F., Scarano, F., 2016. Geometry effect of isolated roughness on boundary layer transition investigated by tomographic PIV. International Journal of Heat and Fluid Flow, vol 61, pp 31-44.

[128] Zhang, Q., Schröder, W., Meinke, M., 2010. A zonal RANS-LES method to determine the flow over a high-lift configuration. Computer \& Fluids, vol 39, pp 1241-1253.

[129] Zhang, Y., Habashi, W., Khurram, R., 2016. Zonal Detached-Eddy Simulation of Turbulent Unsteady Flow over Iced Airfoils. Journal of Aircraft, vol 53, No 1, pp 168-181, doi: 10.2514/1.C033253. 